\title{
How brain reacts to targeted attack at a hub region
}

$3{ }^{1}$ Neuroscience Program, The Huck Institutes of the Life Sciences, The Pennsylvania State

4 University, University Park, PA 16802, USA

$5 \quad{ }^{2}$ Department of Biomedical Engineering, The Pennsylvania State University, University Park,

6 PA 16802, USA

8 *Address for correspondence:

9 Nanyin Zhang, PhD

10 Professor of Biomedical Engineering and Electrical Engineering

11 Lloyd \& Dorothy Foehr Huck Chair in Brain Imaging

12 The Huck Institutes of the Life Sciences

13 The Pennsylvania State University

14 W-341 Millennium Science Complex, University Park, PA 16802, USA

15 Email: nuz2@psu.edu

16

17

Conflict of interest: none.

19

20 Keywords: DREADD, resting-state fMRI, default mode network, graph theory, awake, rat 


\section{Abstract}

The architecture of brain networks has been extensively studied in multiple species.

24 However, exactly how the brain network reconfigures when a local region stops

25 functioning remains elusive. By combining chemogenetics and resting-state functional

26 magnetic resonance imaging (rsfMRI) in awake rodents, we investigated the causal

27 impact of acutely inactivating a hub region (i.e. dorsal anterior cingulate cortex) on brain

28 network properties. We found that disrupting hub activity profoundly changed the function

29 the default-mode network (DMN), and this change was associated with altered DMN-

30 related behavior. Suppressing hub activity also impacted the topological architecture of

31 the whole-brain network in network resilience, segregation and small worldness, but not

32 network integration. This study has established a system that allows for mechanistically

33 dissecting the relationship between local regions and brain network properties. Our data

34 provide direct evidence supporting the hypothesis that acute dysfunction of a brain hub

35 can cause large-scale network changes. This study opens an avenue of manipulating

36 brain networks by controlling hub-node activity. 


\section{Introduction}

The mammalian brain is a highly inter-connected network system with distributed brain regions orchestrating to support normal function and mediate complex behavior (1).

41 The brain network is organized to maintain an optimal balance of information segregation and integration, which simultaneously maximizes the efficiency of distal communication via long-range connections and minimizes the wiring cost through clustered local processing $((2,3)$, but also see (4)). Such organization is well conserved in multiple species including rodents, primates and humans (5-7).

It is becoming increasingly clear that altered brain network organization is tightly

47 linked to brain disorders (8-10). However, the neural substrates causing these large-scale network changes remain unknown. A key hypothesis is the dysfunction of brain hub regions. Hubs are brain regions that have high degrees of connections with the rest of the brain $(11,12)$. Because of their central roles, dysfunction of hub nodes can change global

51 integrative process, and has been hypothesized to be a direct cause of altered brain

52 network properties and pathophysiology of brain disorders (13-15). For instance, brain

53 network analysis in patients with Alzheimer's Disease showed that amyloid-beta

54 deposition mainly accumulated in functional hubs (16). Therefore, comprehensively

55 understanding the causal relationship between hub activity and brain network

56 organization is critical. However, directly testing this hypothesis in humans is challenging,

57 as selectively altering activity in a hub and dissecting its causal impact on brain networks 58 are difficult.

This obstacle can be overcome in experimental animals using neuroscience tools 
61 state functional magnetic resonance imaging (rsfMRI). DREADDs introduce genetically

62 encoded modified muscarinic G-protein-coupled receptors into living neurons, which allow reversible in vivo manipulation of neuronal activity of a defined brain region for hours (17). rsfMRI measures brain-wide resting-state functional connectivity (RSFC), and provides comprehensive assessment of brain network properties $(5,18)$. Thus, combining these two techniques offers a system that allows us to characterize the reconfiguration of the brain network as the neural activity of a network node is selectively perturbed. Given the highly conserved brain network architecture in the mammalian brain (5-7), the results are translatable to humans.

Here we investigate the causal impact of manipulating a hub region, dorsal anterior

71 cingulate cortex (dACC), on brain network function and organization, as well as behavior using an awake rodent model established in our lab (19-22). Imaging awake rodents avoids the confounding effects of anesthesia (23-26), and permits linking imaging to behavioral data $(21,27)$. dACC is selected because it is a known functional and anatomical hub in both human and rat brain $(11,19,28,29)$. We examine the impact of suppressing the dACC on the default mode network (DMN), which is composed of a group rodent DMN (34), manipulating dACC activity provides an avenue for understanding the rodent DMN at the functional level. Our data show that suppressing the dACC disrupts 
activity and connectivity across the whole DMN, and DMN activity changes are correlated with altered DMN-related behaviors. These data suggest that, like humans, DMN in rodents is a functional network with coordinated activity to mediate behavior. Furthermore, we demonstrate that suppressing the dACC impacts the organization of the whole-brain network including network resilience, segregation and small worldness, but not network integration. All these changes are absent in sham rats or when a non-hub region is suppressed. Taken together, this study provides direct measurement of the brain's response to targeted attack at a hub region, and presents a comprehensive framework demonstrating the pivotal role of hubs in the brain network.

\section{Results}

Animals were stereotactically injected with adeno-associated viruses (AAVs) expressing inhibitory G-protein coupled hM4Di receptor with a pan-neuronal synapsin promoter (AAV8.hSyn.hM4Di.mCherry, Addgene). After 4-6 weeks of recovery and protein expression (Fig. S1), animals received either Clozapine-N-Oxide (CNO) or saline 30 min before electrophysiology recording, rsfMRI scanning or behavioral tests. Saline and $\mathrm{CNO}$ sessions were separated by at least 3 days in a random order. The experimental procedure is summarized in Fig. 1.

DREADD suppressed both evoked and spontaneous neural activities in local regions.

We first validated the inhibitory effect of the DREADD using a visual stimulation paradigm (Fig.2). AAVs expressing inhibitory DREADD (AAV8.hSyn.hM4Di.mCherry, Addgene) were injected into the superior colliculus (SC). After recovery and DREADD 
expression, neural activity in the $S C$ was activated by visual stimuli (Figs. 2A-D, 1 flash/trial and 5 flashes/trial, $100 \mathrm{~ms}$ per flash, $10 \mathrm{sec}$ per trial, 15 trials per animal) and measured by electrophysiology. SC firing rates were significantly reduced $30 \mathrm{~min}$ after CNO injection (two-sample tests, 1 flash/trial, $t=10.30, p=8.8 \times 10^{-17}$, Figs. $2 B \quad \& 2 D ; 5$ flashes/trial, $t=13.48, p=4.1 \times 10^{-23}$, Fig. $\left.2 C \& 2 D\right)$. In addition, spontaneous neural activities including spiking activity and local field potential (LFP), quantified by the electrophysiology data $5 \mathrm{~s}$ before the onset of visual stimulation in each trial, were significantly dampened in CNO-injected rats (MUA: $t=16.15, p=1.041 \times 10^{-36} ;$ LFP: $t=$ $5.84, p=8.6 \times 10^{-8} ;$ Figs. $2 \mathrm{E}, 2 \mathrm{~F} \& 2 \mathrm{G}$ ). The LFP spectrograms after saline and CNO injections in a representative rat were also shown in Fig. S2. These data collectively confirmed the inhibitory effect of the DREADD on evoked and spontaneous neural activities in rats.

Suppressing the dACC reduced its rsfMRI signal, RSFC and local network organization.

The bilateral dACC was transfected by inhibitory DREADDs under a pan-neuronal synapsin promoter (AAV8.hSyn.hM4Di.mCherry, Addgene). Sham rats received a control virus (AAV8-hsy-GFP, Addgene). Animals were scanned using rsfMRI twice in the awake state, with each session starting 30 min after either $\mathrm{CNO}$ or saline injections. The two imaging sessions were separated by at least 3 days with a randomized order. There was no difference in motion level during rsfMRI scanning between $\mathrm{CNO}$ and saline injection conditions in both DREADD and sham groups (Fig. S3).

Fig. $3 A$ shows that DREADD suppression of the dACC reduced its resting-state blood-oxygenation-level dependent (BOLD) amplitude (std normalized by mean, $t=2.00$, 
$130 p=0.048)$, again confirming the inhibitory effect of the DREADD on spontaneous neural

131 activity. Suppressing the dACC also affected its functional connectivity, reflected by

132 generally reduced RSFC in dACC seedmaps (for each map: one sample t test, $p<0.0005$,

133 linear mixed model, false discovery rate (FDR) corrected) after CNO injection relative to

134 saline injection (Fig. 3B). We further examined the impact of suppressing the dACC on

135 its local information processing, assessed by the modular structure, which is defined as

136 a group of nodes with significantly greater within-module connections than between-

137 module connections (38). The dACC-related module shrunk in size in DREADD rats after

138 CNO injection relative to saline injection at high graph densities $(p<0.05$, linear mixed

139 model, Fig. 3E), suggesting that suppressing the dACC mainly dropped relatively weaker

140 connections in the dACC module.

141 In addition to dACC-related circuits, we assessed RSFC between regions of

142 interest (ROIs) across the whole brain. Multiple distributed connections exhibited altered

143 RSFC (two-sample t-tests, $p<0.05$, linear mixed model, FDR corrected) with lower RSFC

144 in the majority of these connections (i.e. blue elements in the T map, Figs. 3C \& 3D),

145 indicating that the impact of suppressing neural activity in a hub can go beyond the hub

146 itself and propagate to neural circuits across the whole brain. This ripple effect is

147 consistent with the report in human patients with focal brain lesions to critical locations 148 (39).

None of these changes (i.e. resting-state BOLD amplitude, dACC-related RSFC and community size) were observed in sham rats when comparing $\mathrm{CNO}$ - to saline-

151 injection conditions (Fig. S4), which suggests that CNO had minimal off-target effects in 152 our system, ruling out its potential confounding effects on rsfMRI data (40). 
Suppressing $d A C C$ altered activity and connectivity in the DMN.

Given that $\mathrm{dACC}$ is a major hub node in the functional network of DMN, we examined the impact of inactivating the $\mathrm{AACC}$ on the DMN organization in awake rodents. We first mapped the DMN using fractional amplitude of low-frequency fluctuations (fALFF), defined by voxel-wise low-frequency spectrum power $(0.01-0.08 \mathrm{~Hz})$ normalized by the full-spectrum power (41) of the rsfMRI signal. This well-established method measures the amplitude of regional spontaneous brain activity. Thus, it can reliably identify brain regions with higher activity at rest, and map the spatial pattern of the DMN (41).

Fig. 4 displays the DMN spatial maps in DREADD and sham rats after saline and CNO injections, respectively. Brain regions highlighted in the network included the ACC, posterior cingulate cortex (PCC), prelimbic cortex (PL), retrosplenial cortex (RSC), orbital cortex (ORB), posterior parietal cortex (PPC), as well as subcortical regions of the basal forebrain (BF) and hypothalamus (Hypo). This DMN pattern was reproduced with a completely different analysis method (i.e. independent component analysis, Fig. S5) previously used by other groups, and well agreed with the rodent DMN pattern reported in the literature (34-36). Notably, we found that the BF was a prominent node in the rodent DMN, which was not observed in other rodent DMN mapping studies, but was reported 171 in an electrophysiology study that demonstrated that the BF is a key DMN node and 172 critical in regulating DMN-related behaviors in rats (42).

174 of virtually the entire network (Fig. 4A, top row), reflected by reduced BOLD fALFF in the 175 ACC, dPCC, RSC, PL, PPC, BF and Hypo after CNO injection in DREADD-suppressed 
176 rats (Fig. 4B, top row, $p<0.05$, Family-wise error corrected, and Fig. 4C). Again, no

177 regions displayed reduced spontaneous activity in sham rats after CNO injection (Figs.

178 4A \& 4B, bottom row). We also calculated RSFC between every pair of nodes in the DMN

179 including the dorsal and ventral $\mathrm{ACC}$, dorsal and ventral PCC, RSC, ORB, PL, PPC, as well as BF and Hypo (34) in both DREADD and sham rats after saline and CNO injections,

181 respectively (Fig. 4D). Cortical nodes generally displayed significantly reduced RSFC ( $p$

$182<0.05$, FDR corrected, Figs. 4E \& 4F), except that the RSC exhibited increased RSFC

183 with the PPC, BF and Hypo after CNO injection. In contrast, both subcortical nodes (BF

184 and Hypo) showed increased RSFC after dACC suppression (Figs. 4E \& 4F). Taken

185 together, these data revealed that the DMN was significantly reorganized when dACC

186 activity was suppressed, suggesting that the rodent DMN is a functional network with

187 coordinated activity, and the dACC is a pivotal node in this network.

Suppressing $d A C C$ altered DMN-related behaviors in animals.

Considering that suppressing dACC significantly altered DMN activity in awake

rodents, we hypothesize that it also changes DMN-related behavior, measured by quiet restfulness in rodents. Nair and colleagues demonstrated that quiet restfulness in homecage is characteristic behavior to DMN activity, evidenced by elevated neural activity in the DMN, including ACC and BF, during this behavioral state (42). Robust DMN activation

195 during quiet restfulness was also reported in chimpanzees (43). Therefore, we evaluated 196 quiet restfulness, defined by continuous immobility for at least $2 \mathrm{~s}$, in our animals. After

197 either CNO or saline injection, the rat was put in the home cage for $45 \mathrm{~min}$ and was video198 recorded. The animal's behavior was analyzed using behavioral tracking software (ANY- 
maze, Stoelting Co., Wood Dale, IL). After CNO injection, quiet restfulness was significantly reduced in the rat (Fig. 5), reflected by significantly lower immobile time for at least $2 \mathrm{~s}(\mathrm{t}=3.991, p=0.0009)$, but higher distance travelled $(t=4.294, p=0.0004)$, mean speed $(t=4.811, p=0.0001)$ and total mobile time $(t=3.991, p=0.0009)$. Rats also displayed significantly higher rearing after CNO injection $(t=2.80, p=0.01)$, likely reflecting an increase in vigilance and/or exploratory behavior. Reduced quiet restfulness remained consistent during the last 15 min of testing, suggesting that these behavioral changes were not due to the environmental changes at the beginning of the test (Fig. S6). We also examined whether DMN activity changes could explain altered DMNrelated behaviors. In 8 animals, both behavioral and imaging data were collected after DREADD expression. Notably, both data were collected in the awake state, which permits linking results of these two measures. We found that fALFF changes in the $\mathrm{APCC}$ and PL were significantly correlated to changes in all measures of DMN-related behavior including distance travelled (dPCC: $r=0.80, p=0.016 ; P L: r=0.79, p=0.019$ ), mean speed (dPCC: $r=0.85, p=0.0077 ; P L: r=0.84, p=0.0095)$, mobile time (dPCC: $r=0.74$, $p=0.036 ; P L: r=0.80, p=0.017$ ) and immobile time (dPCC: $r=0.74, p=0.036 ; P L: r=$ $0.80, p=0.017$ ). These data collectively demonstrate that $d A C C$ suppression alters DMN activity, and DMN activity changes can predict alteration in DMN-related behaviors.

\section{Suppressing a hub node changed whole-brain network organization}

We further investigated the effect of suppressing the dACC on the organization of the whole-brain network architecture in four aspects: network resilience, functional segregation, functional integration, and small-worldness. The rat brain was parcellated 
222 into 68 bilateral ROls based on the Swanson atlas (44). The whole-brain network was 223 constructed by calculating RSFC between every two ROIs, generating a RSFC matrix for

224 each animal at each condition. This RSFC matrix was then binarized based on graph

225 density. Graph topological parameters including assortativity, modularity, global efficiency, 226 and small-worldness were respectively calculated as a function of graph density.

Assortativity measures network resilience. Brain networks with high assortativity are more resistant to local attack such as lesion and neurological degeneration $(12,45)$. Fig. 6 shows that suppressing the dACC considerably reduced the network assortativity, and as a consequence, the whole-brain network became more vulnerable (Fig. 6A). Functional segregation, quantified by modularity, measures the network's ability of specialized processing. We observed that dACC suppression decreased the modularity of the global brain network, indicating reduced functional segregation (Fig. 6C). Global 234 efficiency measures functional integration, which assesses the ability to integrate specialized information from distributed brain regions. Interestingly, suppressing the dACC did not seem to affect the global efficiency (Fig. 6D). Small-worldness reflects the ability to balance functional segregation and integration. Suppressing the dACC reduced small-worldness of the whole-brain network (Fig. 6B). In summary, we observed that suppression of the dACC affected global brain topology including network resilience, network segregation and small-worldness, but not network integration.

As expected, DREADD suppression of the dACC significantly lowered its degree

242 (Fig. 7), suggesting that dACC lost its hubness in the network. Interestingly, the degree 243 of the ventral RSC was significantly increased after dACC suppression, which is 244 consistent with our data that some RSC connections increased their RSFC within the 
245 DMN (Figs. 4E \& 4F). Again, no changes in the degree of dACC or vRSC were observed

246 in sham rats (Fig. S7). These results indicate that a new hub can emerge when an existing

247 hub stops functioning, likely due to the compensatory mechanism.

Suppression of a non-hub region did not change whole-brain network properties

To determine whether aforementioned network changes depended on the specific

251 role of the node (i.e. hub versus non-hub), we also suppressed the activity in a non-hub

252 region-primary visual cortex (V1, bilateral) (19) using the same pan-neuronal inhibitory

253 DREADDs. After CNO injection, significantly reduced BOLD amplitude in V1 was

254 observed (Fig. 8A), which confirmed the inhibitory effect of DREADD in V1. However,

255 suppression of V1 did not affect fALFF in the DMN (Fig. S8), nor did it cause any whole-

256 brain topological changes including network resilience, functional segregation, functional

257 integration, or small-worldness (Figs. 8B-8E). Taken together, these data suggest that

258 attack on separate brain regions can have differential impact on brain network properties,

259 and brain networks are particularly vulnerable to targeted attack at hub nodes.

261 Discussion

For over a century, the neuroscience field has been tremendously moved forward by the effort of examining the relationship between damage of specific brain regions and

264 loss of certain brain function. Classical examples include the discovery of the inferior

265 frontal gyrus in speech production by Paul Broca, and the left posterior temporal cortex

266 in language comprehension by Carl Wernicke. Despite this vital advancement,

267 converging evidence suggests that a large number of complex tasks involve numerous 
268 cognitive processes and require integrated activity from multiple brain regions (46).

269 Therefore, brain function should be viewed as a network phenomenon.

270

By combining DREADDs and rsfMRI in an awake rodent model, we have

271 established a system that eables us to manipulate the activity in any brain region, and

272 measure the corresponding changes in brain-wide networks. This system allows for

273 mechanistically dissecting the causal relationship between a (or multiple) local brain

274 region(s) and brain network properties. In this study we specifically investigated the role

275 of a hub region (i.e. dACC) in brain networks. We found that disrupting hub activity

276 profoundly changed the function of the DMN (Figs 4), and this change was associated

277 with altered DMN-related behavior (Fig. 5). It also impacted the topological architecture

278 of the whole-brain network in terms of network resilience, segregation and small

279 worldness, but not network integration (Fig. 6). These data offer direct evidence

280 supporting the hypothesis that acute dysfunction of a brain hub can cause large-scale

281 network and behavioral changes. They also provide a comprehensive framework

282 documenting the differential impact of inactivating a hub versus a non-hub node on 283 network dynamics.

Tight relationship between local regions and brain networks

The activity in local brain regions can be manipulated using tools like

287 chemogenetic, optogenetic, pharmacological and lesion methods. Such perturbations are

288 often used to study the role of a specific brain region in behavior (47). Meanwhile, the 289 capacity of manipulating local brain regions can be tremendously expanded when 290 combining with neuroimaging approaches with a global field of view (e.g. fMRI), as the 
291 causal impact of local region dysfunction on the rest of the interconnected network can

292 be monitored across the whole brain (48). For instance, using DREADDs and rsfMRI,

293 Grayson and colleagues showed that inactivating the amygdala disrupted communication

294 throughout the cortex in anesthetized monkeys (49). This observation echoes human

295 patient studies in which lesions of critical focal brain locations caused widespread

296 disruption of the brain modular structure $(39,50)$. These results collectively suggest tight

297 linkage between regional activity and large-scale network properties, and highlight the

298 importance of combining local perturbations and global network measurement. Uniquely,

299 in our study we specifically targeted a hub region to directly test the hypothesis that

300 dysfunction of hub nodes is a direct cause of altered brain network properties (13-15). In

301 addition, all data were collected in awake animals, which avoids the confounding effects

302 of anesthesia on brain network function, and permits direct association of network

303 features and behavioral changes. This nature also makes the findings translatable to

304 humans, considering numerous reports of large-scale brain network changes in human

305 brain disorders.

306

DREADDs vs lesion

There are several advantages choosing the DREADD over the lesion method for our research purpose. First, DREADDs allow us to specifically and reversibly inhibit a

310 brain region with minimal damage. On the other hand, long-term damage in brain tissue

311 can decrease metabolism and alter local vascular structure. In addition, there can be

312 excessive blood flow in surrounding regions (51). All these factors will impact fMRI data

313 measurement and can confound RSFC results. Long-term lesion studies can also be 
314 compounded by post-lesion neural plasticity changes resulting from the compensatory mechanism (i.e. secondary effects). Furthermore, most psychiatric disorders are linked to loss of function without tissue damage, making the DREADD method a better

317 translatable model.

DMN in rodents - beyond anatomical resemblance with other species one of its hub nodes (i.e. dACC) in awake rodents. DMN activity changes were also linked to altered DMN-related behaviors after dACC suppression. Although the DMN has been extensively studied in humans including its network structure, function and clinical

324 implications, our understanding of the DMN in rodents is limited. The discovery of rodent

DMN was mainly based on the anatomical resemblance of the network structure with the

DMN in humans and primates $(35,36)$, but its functional role in behavior remains unclear.

Nair and colleagues showed that the rat BF exhibited pronounced gamma oscillations during DMN-related behaviors and this activity was suppressed during active exploration of an unfamiliar environment (42), suggesting that the BF might be a key DMN node that regulates DMN activity. They further demonstrated that the BF controls DMN-related behaviors by affecting neural activity in the ACC. These results are highly consistent with our findings. First, we also observed prominent involvement of the BF in the rat DMN at 333 baseline, which was missing in previous rodent DMN mapping studies (34, 35). Notably, 334 the BF has strong connections with the cortex and subcortical regions like Hypo, and 335 these connected regions substantially overlapped with the DMN revealed in our study (52, 336 53). Second, suppressing the dACC considerably dampened the DMN activity. This result 
337 is consistent with the report that DMN hubs are mostly fragile (54). Third, reduced DMNrelated behaviors resulting from dACC suppression were correlated to activity changes in

DMN nodes. Taken together, these results support the concept that, like humans and primates, the DMN in rodents is a functional network with coordinated neural activity from distributed brain regions, and this network might support behavior related to internally

342 oriented brain states.

\section{Hubs and brain network topology}

The topological changes resulting from hub inactivation, including reduced network resilience, segregation and small worldness, are important for our understanding of the role of individual regions in the global brain network organization. A brain network can be modeled as a graph composed of spatially distributed neuronal components as nodes and anatomical or functional associations between components as edges (2, 3). Topological features of the brain graph are critical in understanding inter-areal information exchange in health and disease. Although the architecture of brain networks has been extensively studied in multiple species $(5,55-57)$, it remains elusive whether different types of nodes, such as hubs versus non-hubs, play distinct or similar roles in the topology

354 of the global brain network. Our data revealed that changes in a hub, but not a non-hub, can significantly alter brain network topology. These results provide critical insight into 356 understanding the pathogenesis of neurological and psychiatric disorders, which indicate 357 that altered brain topological properties reported in these brain disorders might start from dysfunction of certain hub nodes (58). Interestingly, our data showed that network integration was intact after dACC inactivation, likely due to the compensatory effect of the 
so-called 'rich club organization' in the rat brain (19). Another possible explanation is that although the $\mathrm{dACC}$ has high centrality, it functions as a provincial hub as opposed to a connector hub (59). These two types of hubs were demonstrated to have differential impact on brain network properties (39), with connector hubs more affecting network integration, and provincial hubs more affecting network segregation. Our result is consistent with the report that the ACC belongs to the "segregation effect" class, as reported in human lesion studies (60). Our data also support the notion that neural network communication does not necessarily involve the shortest paths between nodes (e.g. navigation routing) (61).

\section{Methods and Materials}

Animals.

44 adult male Long-Evans rats (300-500g) were used in the present study. Animals were housed in Plexiglas cages. Food and water were provided ad libitum. The housing room was maintained at an ambient temperature $\left(22-24{ }^{\circ} \mathrm{C}\right)$ with a $12 \mathrm{~h}$ light: $12 \mathrm{~h}$ dark cycle. All experiments were approved by the Pennsylvania State University Institutional Animal Care and Use Committee.

\section{Surgery.}

Aseptic stereotaxic surgery was conducted to infuse the virus to the brain. Rats were anesthetized by intramuscular (IM) injections of ketamine $(40 \mathrm{mg} / \mathrm{kg})$ and xylazine (12 mg/kg). Dexamethasone $(0.5 \mathrm{mg} / \mathrm{kg})$ and Baytril $(2.5 \mathrm{mg} / \mathrm{kg})$ were injected $\mathrm{SQ}$ to prevent tissue inflammation and bacterial infections. During the surgery, respiration was 
controlled by a ventilator with artificial oxygen. The heart rate was monitored by a pulse oximetry (MouseSTAT® Jr., Kent Scientific Corporation), and the body temperature was maintained at $37{ }^{\circ} \mathrm{C}$ with a warming pad (PhysioSuite, Kent Scientific Corporation). The DREADD virus (AAV8-hSyn-hM4Di-mCherry, $1 \mu \mathrm{L}$ at titer $\geq 3 \times 10^{12} \mathrm{vg} / \mathrm{mL}$, Addgene, Watertown, MA) was bilaterally injected into the dACC (coordinates: AP $+2, M L+/-0.5$, DV -1) and the primary visual cortex (coordinates: AP -6.5, ML +/-3.5, DV -1) for the hub $(n=25)$ and non-hub $(n=8)$ groups, respectively ${ }^{24}$. Sham rats were injected with a control virus (AAV8-hsy-GFP, $1 \mu \mathrm{L}$ at titer $\geq 3 \times 10^{12} \mathrm{vg} / \mathrm{mL}$, Addgene, Watertown, MA, $\mathrm{n}$ $=8)^{24}$ into the dACC. In addition, separate animals used in the visual stimulation experiment were unilaterally injected with AAV8-hSyn-hM4Di-mCherry $(1 \mu \mathrm{L}$ at titer $\geq$ $3 \times 10^{12} \mathrm{vg} / \mathrm{mL}$, Addgene, Watertown, MA, $\mathrm{n}=3$ ) in the SC (coordinates: AP -7, ML +1.5, DV -3). Rats were allowed to recover for at least four weeks.

\section{fMRI experiments:}

Before imaging, animals were acclimated to the MRI environment for 7 days in order to minimize stress and motion during imaging. To better adapt the animal to the restrainer and scanning environment, the acclimation period was gradually increased from 15 min on Day 1 to 30 min on Day 2, 45 min on Day 3, and maintained at 60 min/day for Days 4-7. Detailed procedures for acclimation can be found from our previous publications $(62,63)$. A similar approach to imaging awake animals has also been adopted by other research groups in multiple species (64-66). CNO $(1 \mathrm{mg} / \mathrm{kg}$ in saline, dissolved in DMSO, Sigma-Aldrich, St. Louis, MO) or saline (with DMSO) was IP injected 30 min before rsfMRI data acquisition, at a random order with at least three days apart. 
All imaging sections were conducted at the High Field MRI Facility at the 407 Pennsylvania State University on a 7T Bruker 70/30 BioSpec running ParaVision 6.0.1

408 (Bruker, Billerica, MA). rsfMRI data were acquired at the awake state using T2*-weighted 409 gradient-echo rsfMRI images using the echo-planar-imaging (EPI) sequence with the 410 following parameters: repetition time $=1000 \mathrm{~ms}$; echo time $=15 \mathrm{~ms}$; matrix size $=64 \times 64$;

411 field of view $=3.2 \times 3.2 \mathrm{~cm}^{2}$; slice number $=20$; slice thickness $=1 \mathrm{~mm}$; flip angle $=60$;

412600 volume each run. Three runs were acquired each rsfMRI session. Raw EPI images 413 were shown in Fig. S9.

Data analysis:

All acquired image data were aligned to a defined atlas using Medical Image

417 Visualization and Analysis software (MIVA, http://ccni.wpi.edu/), and then subjected to 418 motion correlation (SPM12), spatial smoothing (Gaussian kernel, full-width at half419 maximum $=1 \mathrm{~mm}$ ), voxel-wise nuisance regression of motion parameters, as well as the 420 signals from the white matter and ventricles, and bandpass filtering $(0.01-0.1 \mathrm{~Hz})$. Before 421 these steps, rsfMRI volumes with relative framewise displacement (FD) $>0.25 \mathrm{~mm}$ and 422 the preceding and following volumes were removed. In addition, the first 10 volumes of 423 each rsfMRI scan were discarded to ensure steady state of magnetization. rsfMRI scans 424 with $>15 \%$ volumes discarded were removed from further analysis.

fALFF was determined by calculating the ratio of the power in the low-frequency range $(0.01-0.08 \mathrm{~Hz})$ to that of the full spectrum $(0-0.25 \mathrm{~Hz})$. For each voxel, the time

427 course was transformed to the frequency domain using FFT. We calculated the ratio 428 between the square root of power in the range of $0.01-0.08 \mathrm{~Hz}$ and that across the entire 
429 frequency range. fALFF changes after DREADD inhibition were obtained using voxel-

430 wise two-sample t-tests with family wise error correction $(P<0.05$, FWE corrected)

431 between data acquired after CNO injection versus saline injection.

Seed RSFC maps were generated using seed-based correlation analysis. Pearson

cross-correlation coefficients between the regionally averaged time course from all voxels

434 inside the seed and time courses of individual voxels were calculated. The corresponding

435 correlation coefficients were Fisher transformed, averaged across all scans and animals

436 in each group, and transformed back to r values.

The whole-brain RSFC network was constructed by parcellating the rat brain into

functional systems including amygdala complex, striatum, hypothalamus, hippocampus,

441 retro-hippocampal regions. RSFC between each pair of ROls was calculated by Pearson

442 correlation of regionally averaged time series of the ROls.

A hub score of each node ranged from 0-4 was determined by the number of the

444 following four criteria the node met: (1) $20 \%$ highest degree; (2) $20 \%$ highest

445 betweenness centrality; (3) 20\% lowest characteristic path length; (4) $20 \%$ lowest local 446 clustering coefficient.

447 Topological parameters of the whole-brain network including the modularity, global

448 efficiency, small-worldness and assortativity were calculated on binarized network across

449 the graph density ranging from 0.15 to 0.45 with a step of 0.05 using the brain connectivity

450 toolbox $(\mathrm{BCT})^{30}$ based on the following equations:

451 Assortativity (45): 


$$
r=\frac{l^{-1} \sum_{(x, y \in L)} k_{x} k_{y}-\left[l^{-1} \sum_{(x, y \in L)} \frac{1}{2}\left(k_{x}+k_{y}\right)\right]^{2}}{l^{-1} \sum_{(x, y \in L)} \frac{1}{2}\left(k_{x}^{2}+k_{y}^{2}\right)-\left[l^{-1} \sum_{(x, y \in L)} \frac{1}{2}\left(k_{x}+k_{y}\right)\right]^{2}}
$$

where $\mathrm{k}_{\mathrm{x}}, \mathrm{k}_{\mathrm{y}}$ is the degree of node $\mathrm{x}$ and $\mathrm{y}$ on two opposite ends of an edge; $\mathrm{L}$ is the set

454 of all edges within the network, and I is the total number of edges.

Modularity (67):

459 where $M$ represents a set of non-overlapping modules, and $\mathrm{e}_{\mathrm{ij}}$ is the proportion of 460 between-modules connections of module $\mathrm{i}$ and $\mathrm{j} ; \mathrm{e}_{\mathrm{ii}}$ is the proportion of within-modules 461 connections of module i.

462 Global efficiency (68):

$$
Q=\sum_{i \in M}\left[e_{i i}-\left(\sum_{j \in M} e_{i j}\right)^{2}\right]
$$

cra

where $\mathrm{n}$ is the total number of nodes; $\mathrm{N}$ is the set of all nodes. $\mathrm{d}_{\mathrm{xy}}$ is the shortest path length between nodes $\mathrm{x}$ and $\mathrm{y}$.

466 Small-worldness (69):

$$
E=\frac{1}{n} \sum_{i \in N} \frac{\sum_{y \in N, y \neq x} d_{x y}^{-1}}{n-1}
$$

468 where $\mathrm{C}$ and $\mathrm{C}_{\text {rand }}$ are the clustering coefficients of the testing network and a random 469 network, respectively; $L$ and $L_{\text {rand }}$ are the characteristic path lengths of the testing 470 network and a random network.

471 At each graph density, all topological parameters were determined for each scan. 472 Changes in each parameter was determined by two-sample t-tests (linear mixed model) 
473 between the CNO injection and saline injection conditions.

474

475

476

477

478

479

480

481

482

483

484

485

486

487

488

489

490

491

492

493

494

495

\section{Behavioral Test}

Animals underwent two behavioral sessions with either $\mathrm{CNO}$ or saline injection with a random order separated by at least 7 days. Each session was composed of a 45 min home-cage test. Before the test, the animal was acclimated to the behavioral room for $15 \mathrm{~min}$, followed by either CNO $(1 \mathrm{mg} / \mathrm{kg})$ or saline injection. The animal was then put back to its home-cage in the behavioral room for $45 \mathrm{~min}$. Behaviors including the total distance traveled, mean speed, time of mobility and time of immobility for at least 2s were recorded by an infrared camera and analyzed by behavioral tracking software (ANY-maze, Stoelting Co., Wood Dale, IL).

\section{Electrophysiology.}

Electrophysiology recordings were conducted in animals with inhibitory DREADD expressed in the SC. Rats were initially anesthetized by IM injections of ketamine (40 $\mathrm{mg} / \mathrm{kg}$ ) and xylazine (12 mg/kg). An electrode (Neuronexus, Ann Arbor, MI) was then inserted into the SC to measure visually-evoked neuronal response to light stimulation produced by a blue laser source (100 mW, $473 \mathrm{~nm}$, Opto Engine) coupled with an optic fiber, which was placed $5 \mathrm{~cm}$ away from the contralateral eye. Before recording, rats were injected with either saline or $\mathrm{CNO}$ in each session. During recording, light anesthesia was maintained using isoflurane ( $0.75 \%)$. Each session included 15 trials of visual stimulation, presented as a single light flash (100 ms per flash) or five flashes (100 ms each flash, $100 \mathrm{~ms}$ inter-flash interval) every $10 \mathrm{~s}$. Light stimulation was controlled by a 
496 custom Labview program. The electrophysiological signal was amplified and sampled at

$49725 \mathrm{kHz}$ using a Neuronexus recording system (Neuronexus, Ann Arbor, MI). Raw data

498 were bandpass filtered (MUA: 300-3000 Hz, LFP: 3-300 Hz). Spikes larger than three

499 times of the standard deviation of electrophysiological signal were detected and clustered

500 at a bin size of $50 \mathrm{~ms}$ in peristimulus-timed histograms (PSTHs).

501

502 Histology.

503 After electrophysiology, animal was perfused with saline followed by $4 \%$ PFA 504 solution. The brain was taken out carefully and stored in solution with $4 \%$ 505 paraformaldehyde and $20 \%$ sucrose. After fixation, the brain was sliced into $60 \mu \mathrm{m}$ slices. 506 Fluorescent expression in the injection site was examined under microscopy.

Acknowledgments

We would like to thank Yikang Liu and David Dopfel for their technical support. The 510 present study was partially supported by National Institute of Neurological Disorders and 511 Stroke Grant R01NS085200 (PI: Nanyin Zhang, PhD) and National Institute of Mental 512 Health Grant R01MH098003 and RF1MH114224 (PI: Nanyin Zhang, PhD).

514 Author Contributions: N.Z. designed the research; W.T., Z.M. and Y.M. performed 515 animal experiments; W.T. and Y.M. performed data analysis; N.Z. and W.T. wrote the 516 paper.

517

518 Conflict of interest: the Authors declare no competing interests. 
Data availability: Data supporting the findings of this manuscript are available from the corresponding author upon reasonable request.

522

\section{References:}

524

1. E. T. Bullmore, D. S. Bassett, Brain graphs: graphical models of the human brain connectome. Annu Rev Clin Psychol 7, 113-140 (2011).

2. E. Bullmore, O. Sporns, Complex brain networks: graph theoretical analysis of structural and functional systems. Nat Rev Neurosci 10, 186-198 (2009).

3. E. Bullmore, O. Sporns, The economy of brain network organization. Nat Rev Neurosci 13, 336-349 (2012).

4. R. F. Betzel, D. S. Bassett, Specificity and robustness of long-distance connections in weighted, interareal connectomes. Proc Natl Acad Sci U S A 115, E4880-E4889 (2018).

5. Z. Liang, J. King, N. Zhang, Uncovering intrinsic connectional architecture of functional networks in awake rat brain. J Neurosci 31, 3776-3783 (2011).

6. J. L. Vincent et al., Intrinsic functional architecture in the anaesthetized monkey brain. Nature 447, 83-86 (2007).

7. J. Wang, X. Zuo, Y. He, Graph-based network analysis of resting-state functional MRI. Front Syst Neurosci 4, 16 (2010).

8. Y. Liu et al., Disrupted small-world networks in schizophrenia. Brain 131, 945-961 (2008).

9. D. S. Bassett, E. T. Bullmore, Human brain networks in health and disease. Curr Opin Neurol 22, 340-347 (2009).

10. A. Fornito, A. Zalesky, M. Breakspear, The connectomics of brain disorders. Nat Rev Neurosci 16, 159-172 (2015).

11. M. P. van den Heuvel, O. Sporns, Network hubs in the human brain. Trends Cogn Sci 17, 683-696 (2013).

12. M. Rubinov, O. Sporns, Complex network measures of brain connectivity: uses and interpretations. Neuroimage 52, 1059-1069 (2010).

13. N. A. Crossley et al., The hubs of the human connectome are generally implicated in the anatomy of brain disorders. Brain 137, 2382-2395 (2014).

14. J. Alstott, M. Breakspear, P. Hagmann, L. Cammoun, O. Sporns, Modeling the impact of lesions in the human brain. PLoS Comput Biol 5, e1000408 (2009).

15. T. Takahashi et al., Progressive gray matter reduction of the superior temporal gyrus during transition to psychosis. Arch Gen Psychiatry 66, 366-376 (2009).

16. R. L. Buckner et al., Cortical hubs revealed by intrinsic functional connectivity: mapping, assessment of stability, and relation to Alzheimer's disease. J Neurosci 29, 1860-1873 (2009). 
17. B. N. Armbruster, X. Li, M. H. Pausch, S. Herlitze, B. L. Roth, Evolving the lock to fit the key to create a family of $G$ protein-coupled receptors potently activated by an inert ligand. Proc Natl Acad Sci U S A 104, 5163-5168 (2007).

18. B. Biswal, F. Z. Yetkin, V. M. Haughton, J. S. Hyde, Functional connectivity in the motor cortex of resting human brain using echo-planar MRI. Magn Reson Med 34, 537-541 (1995).

19. Z. Ma et al., Functional atlas of the awake rat brain: A neuroimaging study of rat brain specialization and integration. Neuroimage 170, 95-112 (2018).

20. N. Zhang et al., Mapping resting-state brain networks in conscious animals. $J$ Neurosci Methods 189, 186-196 (2010).

21. D. Dopfel et al., Individual variability in behavior and functional networks predicts vulnerability using an animal model of PTSD. Nat Commun 10, 2372 (2019).

22. Z. Ma, N. Zhang, Temporal transitions of spontaneous brain activity. eLife 7, (2018).

23. Y. R. Gao et al., Time to wake up: Studying neurovascular coupling and brain-wide circuit function in the un-anesthetized animal. Neuroimage, (2016).

24. C. Hamilton, Y. Ma, N. Zhang, Global reduction of information exchange during anesthetic-induced unconsciousness. Brain structure \& function 222, 3205-3216 (2017).

25. Z. Liang, J. King, N. Zhang, Intrinsic organization of the anesthetized brain. $J$ Neurosci 32, 10183-10191 (2012).

26. Y. Ma, C. Hamilton, N. Zhang, Dynamic Connectivity Patterns in Conscious and Unconscious Brain. Brain connectivity 7, 1-12 (2017).

27. Z. Liang, J. King, N. Zhang, Neuroplasticity to a single-episode traumatic stress revealed by resting-state fMRI in awake rats. Neuroimage 103, 485-491 (2014).

28. M. P. van den Heuvel, L. H. Scholtens, M. A. de Reus, Topological organization of connectivity strength in the rat connectome. Brain structure \& function 221, 17191736 (2016).

29. G. Bush, P. Luu, M. I. Posner, Cognitive and emotional influences in anterior cingulate cortex. Trends Cogn Sci 4, 215-222 (2000).

30. D. A. Gusnard, M. E. Raichle, M. E. Raichle, Searching for a baseline: functional imaging and the resting human brain. Nat Rev Neurosci 2, 685-694 (2001).

31. M. E. Raichle et al., A default mode of brain function. Proc Natl Acad Sci U S A 98, 676-682 (2001).

32. D. Zhang, M. E. Raichle, Disease and the brain's dark energy. Nat Rev Neurol 6, 15-28 (2010).

33. S. Whitfield-Gabrieli, J. M. Ford, Default mode network activity and connectivity in psychopathology. Annu Rev Clin Psychol 8, 49-76 (2012).

34. L. M. Hsu et al., Constituents and functional implications of the rat default mode network. Proc Natl Acad Sci U S A 113, E4541-4547 (2016). 
35. H. Lu et al., Rat brains also have a default mode network. Proc Natl Acad Sci U S A 109, 3979-3984 (2012).

36. J. M. Stafford et al., Large-scale topology and the default mode network in the mouse connectome. Proc Natl Acad Sci U S A 111, 18745-18750 (2014).

37. Z. C. Zhou et al., Resting state network topology of the ferret brain. Neuroimage 143, 70-81 (2016).

38. M. E. Newman, Modularity and community structure in networks. Proc Natl Acad Sci U S A 103, 8577-8582 (2006).

39. C. Gratton, E. M. Nomura, F. Perez, M. D'Esposito, Focal brain lesions to critical locations cause widespread disruption of the modular organization of the brain. Journal of cognitive neuroscience 24, 1275-1285 (2012).

40. J. L. Gomez et al., Chemogenetics revealed: DREADD occupancy and activation via converted clozapine. Science 357, 503-507 (2017).

41. Q. H. Zou et al., An improved approach to detection of amplitude of low-frequency fluctuation (ALFF) for resting-state fMRI: fractional ALFF. J Neurosci Methods 172, 137-141 (2008).

42. J. Nair et al., Basal forebrain contributes to default mode network regulation. Proc Natl Acad Sci U S A 115, 1352-1357 (2018).

43. S. K. Barks, L. A. Parr, J. K. Rilling, The default mode network in chimpanzees (Pan troglodytes) is similar to that of humans. Cereb Cortex 25, 538-544 (2015).

44. L. W. Swanson, Brain Maps: Structure of the Rat Brain., (Elsevier, 2004).

45. M. E. Newman, Assortative mixing in networks. Phys Rev Lett 89, 208701 (2002).

46. R. Liegeois et al., Resting brain dynamics at different timescales capture distinct aspects of human behavior. Nat Commun 10, 2317 (2019).

47. P. D. Whissell, S. Tohyama, L. J. Martin, The Use of DREADDs to Deconstruct Behavior. Front Genet 7, 70 (2016).

48. J. H. Lee et al., Global and local fMRI signals driven by neurons defined optogenetically by type and wiring. Nature 465, 788-792 (2010).

49. D. S. Grayson et al., The Rhesus Monkey Connectome Predicts Disrupted Functional Networks Resulting from Pharmacogenetic Inactivation of the Amygdala. Neuron 91, 453-466 (2016).

50. D. E. Warren et al., Network measures predict neuropsychological outcome after brain injury. Proc Natl Acad Sci U S A 111, 14247-14252 (2014).

51. C. Rorden, H. O. Karnath, Using human brain lesions to infer function: a relic from a past era in the fMRI age? Nat Rev Neurosci 5, 813-819 (2004).

52. M. R. Gielow, L. Zaborszky, The Input-Output Relationship of the Cholinergic Basal Forebrain. Cell Rep 18, 1817-1830 (2017).

53. L. J. Agostinelli, J. C. Geerling, T. E. Scammell, Basal forebrain subcortical projections. Brain structure \& function 224, 1097-1117 (2019). 
54. L. L. Gollo et al., Fragility and volatility of structural hubs in the human connectome. Nat Neurosci 21, 1107-1116 (2018).

55. A. Liska, A. Galbusera, A. J. Schwarz, A. Gozzi, Functional connectivity hubs of the mouse brain. Neuroimage 115, 281-291 (2015).

56. A. M. Belcher et al., Large-scale brain networks in the awake, truly resting marmoset monkey. J Neurosci 33, 16796-16804 (2013).

57. D. S. Bassett, E. Bullmore, Small-world brain networks. Neuroscientist 12, 512523 (2006).

58. W. W. Seeley, R. K. Crawford, J. Zhou, B. L. Miller, M. D. Greicius, Neurodegenerative diseases target large-scale human brain networks. Neuron 62 , 42-52 (2009).

59. R. Guimera, L. A. Nunes Amaral, Functional cartography of complex metabolic networks. Nature 433, 895-900 (2005).

60. B. Yuan et al., Brain hubs in lesion models: Predicting functional network topology with lesion patterns in patients. Sci Rep 7, 17908 (2017).

61. C. Seguin, M. P. van den Heuvel, A. Zalesky, Navigation of brain networks. Proc Natl Acad Sci U S A 115, 6297-6302 (2018).

62. D. Dopfel, N. Zhang, Mapping stress networks using functional magnetic resonance imaging in awake animals. Neurobiol Stress 9, 251-263 (2018).

63. Z. Liang, J. King, N. Zhang, Anticorrelated resting-state functional connectivity in awake rat brain. Neuroimage 59, 1190-1199 (2012).

64. E. Bergmann, G. Zur, G. Bershadsky, I. Kahn, The Organization of Mouse and Human Cortico-Hippocampal Networks Estimated by Intrinsic Functional Connectivity. Cereb Cortex 26, 4497-4512 (2016).

65. K. Yoshida et al., Physiological effects of a habituation procedure for functional MRI in awake mice using a cryogenic radiofrequency probe. J Neurosci Methods 274, 38-48 (2016).

66. P. C. Chang et al., Novel method for functional brain imaging in awake minimally restrained rats. J Neurophysiol 116, 61-80 (2016).

67. M. E. Newman, Fast algorithm for detecting community structure in networks. Phys Rev E Stat Nonlin Soft Matter Phys 69, 066133 (2004).

68. V. Latora, M. Marchiori, Efficient behavior of small-world networks. Phys Rev Lett 87, 198701 (2001).

69. M. D. Humphries, K. Gurney, Network 'small-world-ness': a quantitative method for determining canonical network equivalence. PLoS One 3, e0002051 (2008). 
673 Figure Captions.

674 Figure 1. Schematic diagram of the experimental paradigm.

675 Figure 2. Validation of the inhibitory effect of DREADDs. A) Visual stimulation paradigm;

676 Spiking activity in the SC in response to B) 1 flash (100ms/flash) and C) 5 flashes

677 (100ms/flash) of light stimulation after saline and CNO injections (15 trials per animal, per

678 condition, number of animals = 3); D) DREADD suppressed evoked firings (two-sample 679 tests, 1 flash/trial, $t=10.30, p=8.8 \times 10^{-17} ; 5$ flashes/trial, $t=13.48, p=4.1 \times 10^{-23}$ ); E)

680 DREADD suppressed spontaneous firings during 5 s before visual stimulation $(t=16.15$, $\left.681 \mathrm{p}=1.041 \times 10^{-36}\right) ;$ F) Averaged spectrograms after saline (left) and CNO (right) injections; G) Chemogenetic inhibition reduced local field potential power $(3-300 \mathrm{~Hz})$ in the SC $(\mathrm{t}=$ $\left.5.84, p=8.6 \times 10^{-8}\right){ }^{* * *}: p<0.005$

Figure 3. Suppressing $d A C C$ reduced its spontaneous BOLD signal, RSFC, and local network structure. A) Amplitude of spontaneous BOLD activity in DREADD (19 animals, 686 78 scans) and sham groups (8 animals, 41scans); B) Averaged seedmaps after saline and CNO injections (for each map: one-sample t-test, linear mixed model, $p<0.0005$, FDR corrected); C) Left: averaged ROI-wise RSFC matrices after saline and CNO injections in the DREADD group. Right: connections exhibiting significant RSFC change

690 (two-sample t-test, linear mixed model, $p<0.05$, FDR corrected). D) These connections 691 were grouped into multiple brain systems. Line thickness represents the total number of 692 connections with significant RSFC changes between the two systems; E) The size of the 693 dACC-associated community structure as a function of graph density in the DREADD 694 group after saline and CNO injections (two-sample t-test, linear mixed model). *: $p<0.05$; $695 * *: p<0.01$ 
Figure 4. Suppressing $d A C C$ disrupted activity across the DMN. A) DMN constructed using fractional amplitude of low-frequency fluctuations (fALFF) after saline and CNO injections; B) Voxel-wise fALFF changes after DREADD inhibition $(p<0.05$, linear mixed model, family-wise error corrected); C) ROI-wise fALFF changes after DREADD inhibition $\left({ }^{*}: p<0.05 ;{ }^{* *}: p<0.01 ;{ }^{* * *}: p<0.005 ; \#: p=0.11\right)$. D) RSFC between DMN regions in the DREADD and sham groups after saline and CNO injections, respectively; E) RSFC changes between DMN nodes after dACC inhibition $(p<0.05$, linear mixed model, FDR corrected); F) Negative and positive RSFC changes after dACC inhibition $(p<0.05$, linear mixed model, FDR corrected).

Figure 5. Suppressing $d A C C$ changed DMN-related behavior ( $n=14)$. A) Total distance traveled in homecage (45 min); B) Mean speed; C) Total mobile time; D) Total immobile time for at least 2 s. E) Total rearing behavior. ${ }^{*}: p<0.05 ;{ }^{* * *}: p<0.005$. F) Correlations of dPCC activity and DMN-related behaviors. G) Correlations of PL activity and DMNrelated behaviors.

Figure 6. Suppressing a hub node changed whole-brain network topology. A) Network resilience; B) Small-worldness; C) Network segregation; D) Network integration. Linear mixed model; DREADD: $\mathrm{n}=19,78$ scans; sham: $\mathrm{n}=8,41$ scans. ${ }^{*}: \mathrm{p}<0.05 ;{ }^{* *}: \mathrm{p}<0.01$.

Figure 7. New hub emerged after the inactivation of an existing hub. A) Hub scores without and with dACC inactivation, respectively; B) The degree of dACC and ventral RSC without and with dACC inactivation (linear mixed model; $n=19,78$ scans). ${ }^{*}: p<$ $0.05 ;^{* *}: p<0.01 ;^{* * *}: p<0.005$ 
717 Figure 8. Suppressing a non-hub node did not change whole-brain network topology. A) 718 Amplitude of spontaneous BOLD activity in the primary visual cortex after CNO and saline

719 injections ( $p<0.05, n=8,66$ scans); B) Network resilience; C) Small-worldness; D)

720 Network segregation; E) Network integration. 


\section{Figures.}

723

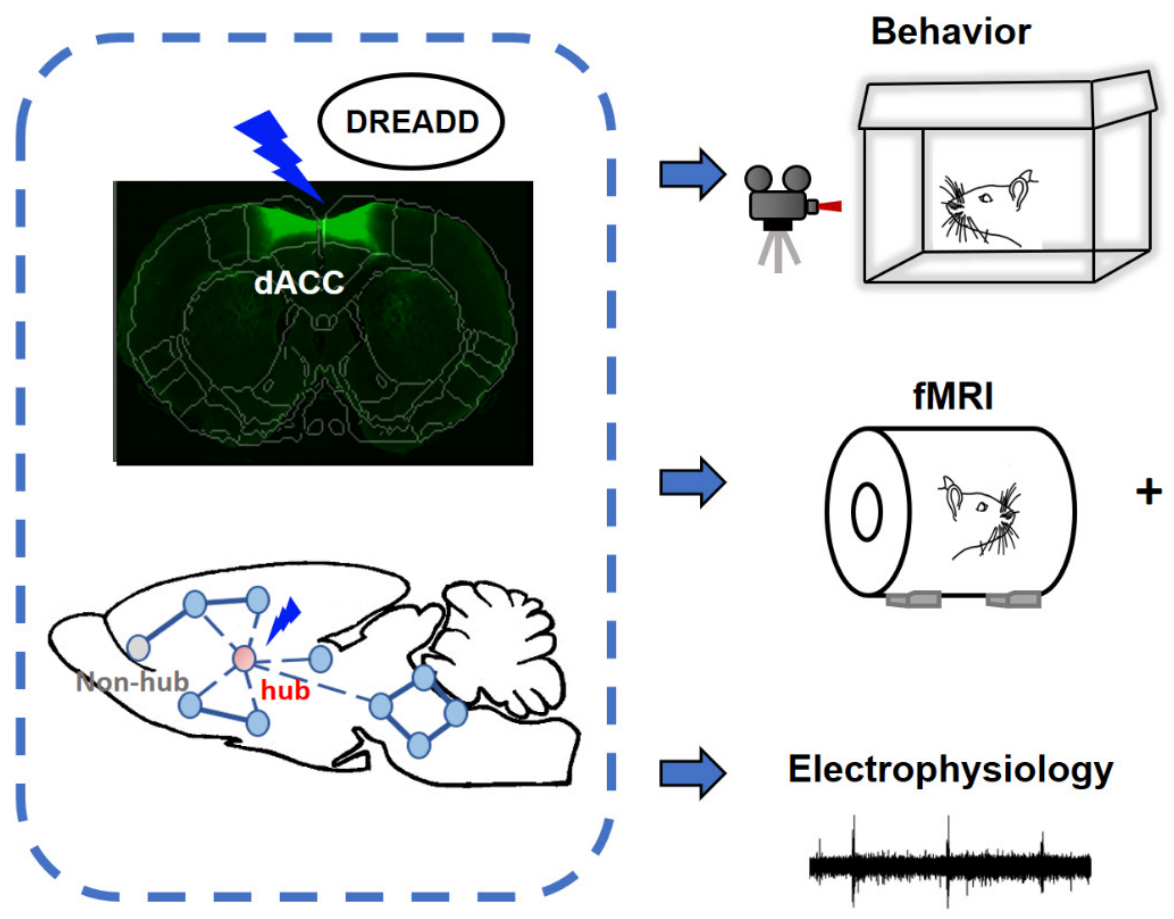

725 Figure 1. Schematic diagram of the experimental paradigm. 

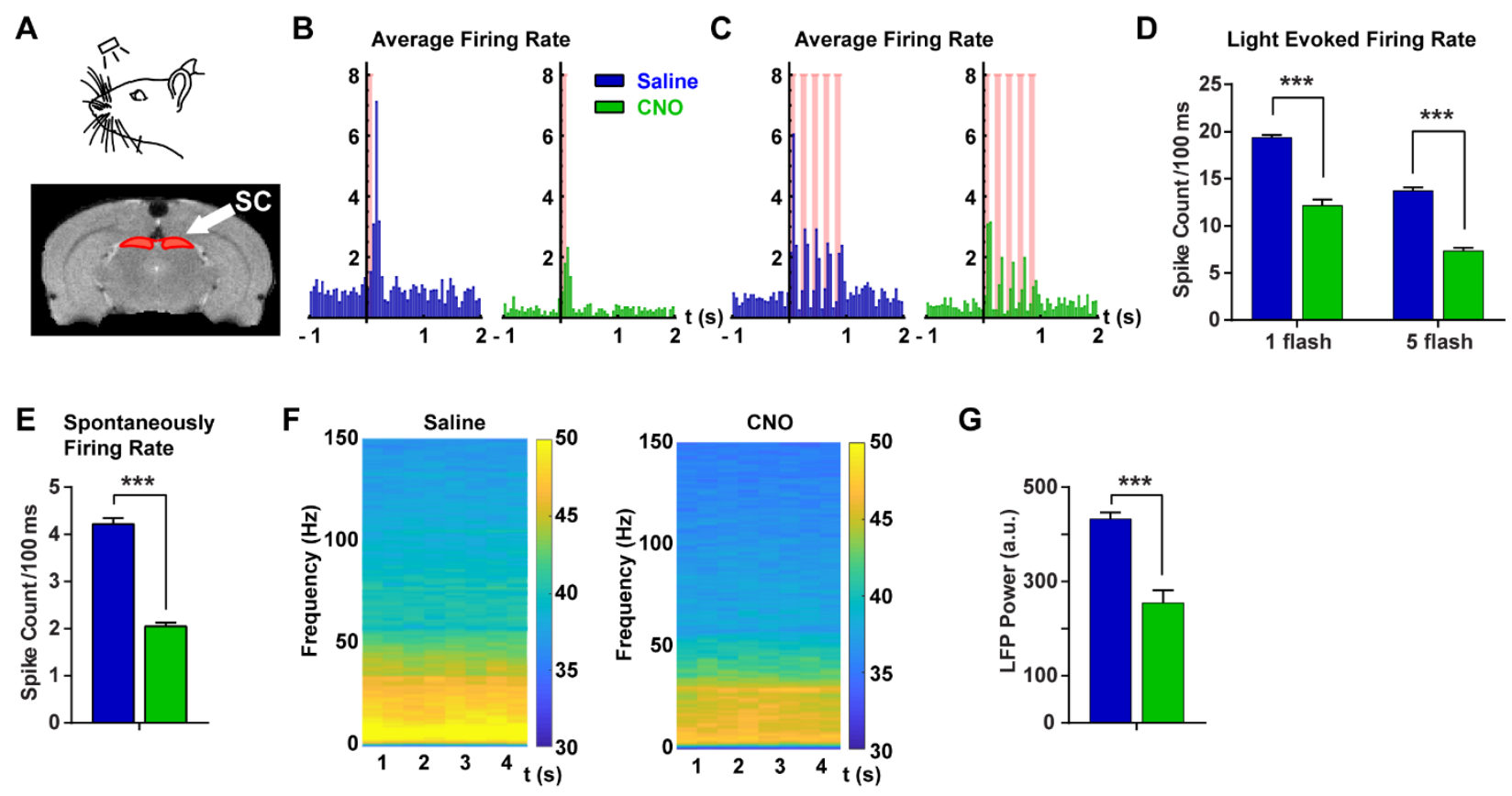

G

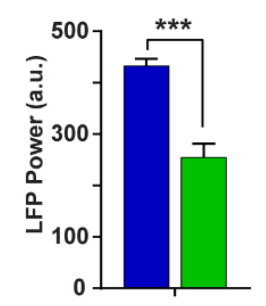

Figure 2. Validation of the inhibitory effect of DREADDs. A) Visual stimulation paradigm; Spiking activity in the SC in response to B) 1 flash $(100 \mathrm{~ms} /$ flash) and C) 5 flashes (100ms/flash) of light stimulation after saline and CNO injections (15 trials per animal, per condition, number of animals = 3); D) DREADD suppressed evoked firings (two-sample tests, 1 flash/trial, $t=10.30, p=8.8 \times 10^{-17} ; 5$ flashes/trial, $\left.t=13.48, p=4.1 \times 10^{-23}\right) ; E$ ) DREADD suppressed spontaneous firings during 5 s before visual stimulation $(t=16.15$, $\mathrm{p}=1.041 \times 10^{-36}$ ); F) Averaged spectrograms after saline (left) and CNO (right) injections; G) Chemogenetic inhibition reduced local field potential power $(3-300 \mathrm{~Hz})$ in the $\mathrm{SC}(\mathrm{t}=$ $\left.5.84, p=8.6 \times 10^{-8}\right){ }^{* * *}: p<0.005$. 
A $\square$ Saline $\square$ CNO

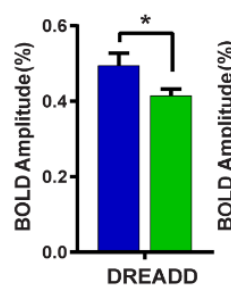

C

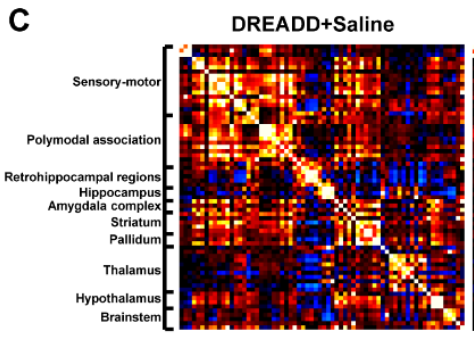

E

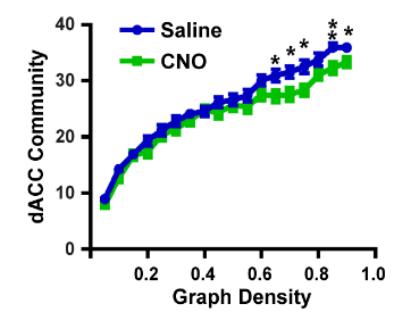

B

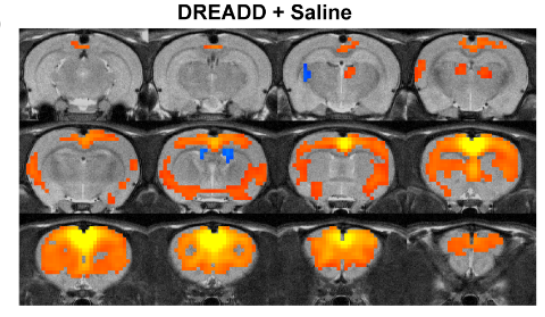

DREADD+CNO

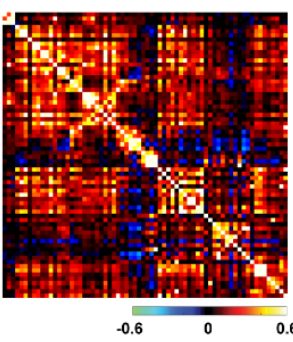

T Map

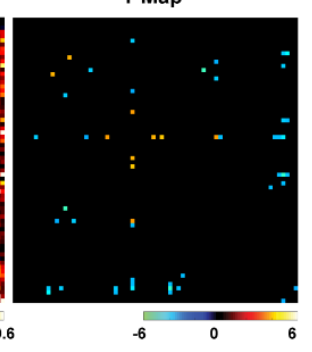

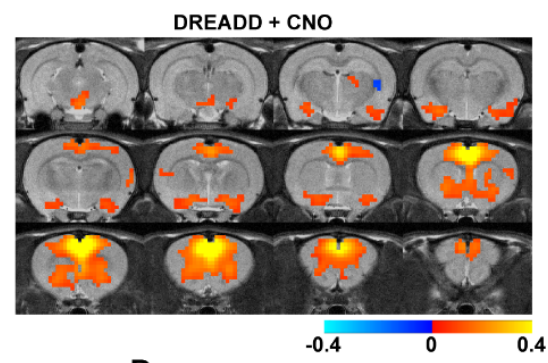

D

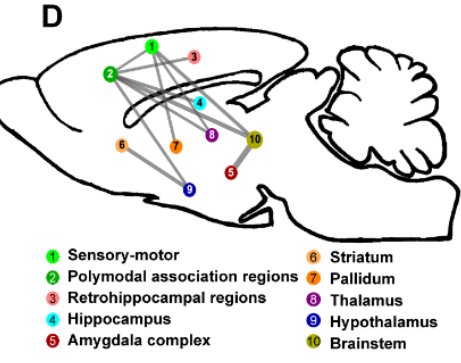

Figure 3. Suppressing $d A C C$ reduced its spontaneous BOLD signal, RSFC, and local network structure. A) Amplitude of spontaneous BOLD activity in DREADD (19 animals, 78 scans) and sham groups (8 animals, 41scans); B) Averaged seedmaps after saline and $\mathrm{CNO}$ injections (for each map: one-sample t-test, linear mixed model, $p<0.0005$, FDR corrected); C) Left: averaged ROI-wise RSFC matrices after saline and CNO injections in the DREADD group. Right: connections exhibiting significant RSFC change (two-sample t-test, linear mixed model, $p<0.05$, FDR corrected). D) These connections were grouped into multiple brain systems. Line thickness represents the total number of connections with significant RSFC changes between the two systems; E) The size of the group after saline and CNO injections (two-sample t-test, linear mixed model). *: $p<0.05$; ${ }^{* *}: p<0.01$. 
A
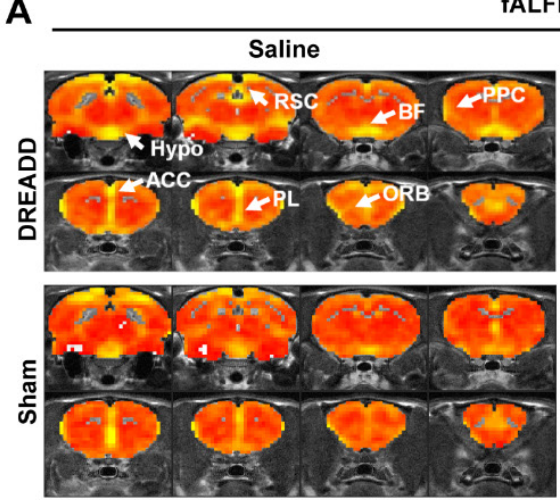

C

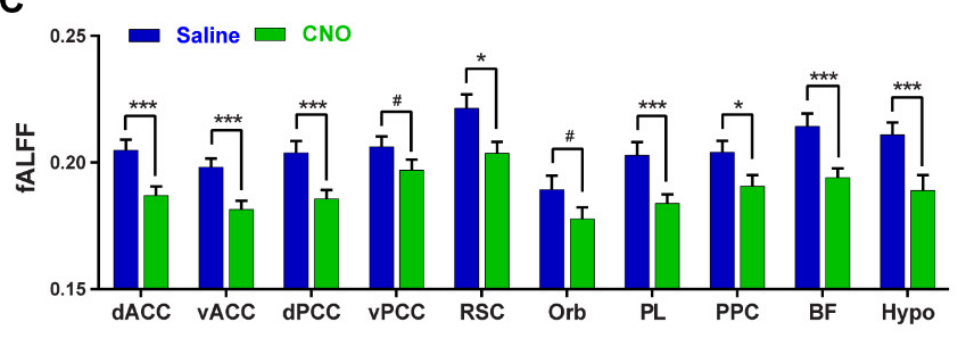

D Saline
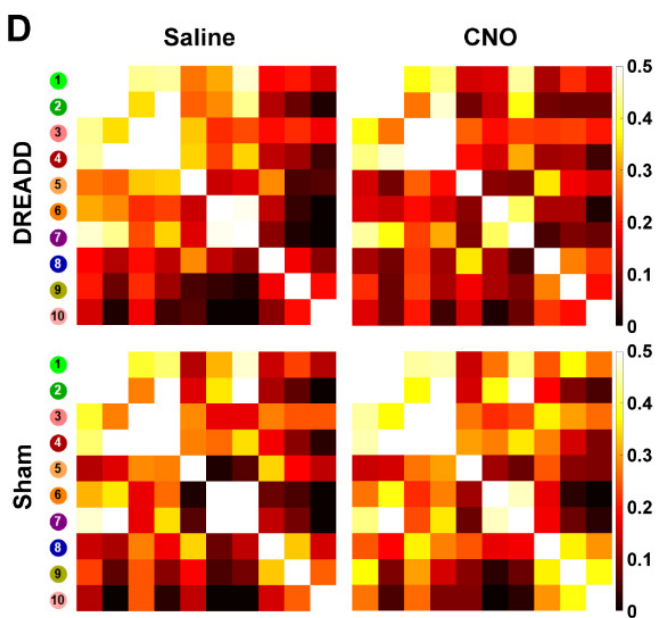

E T Map
fALFF Map

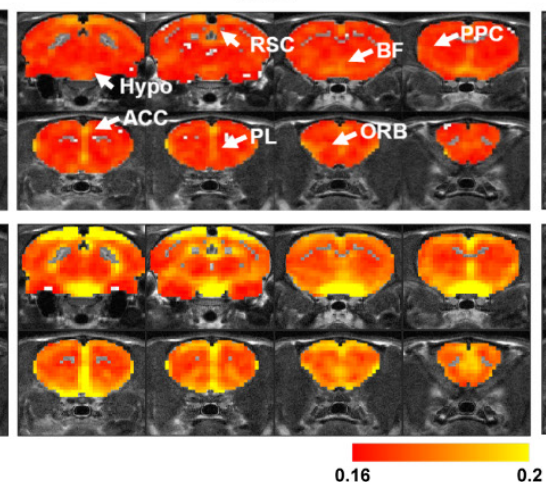

0.16

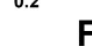

B
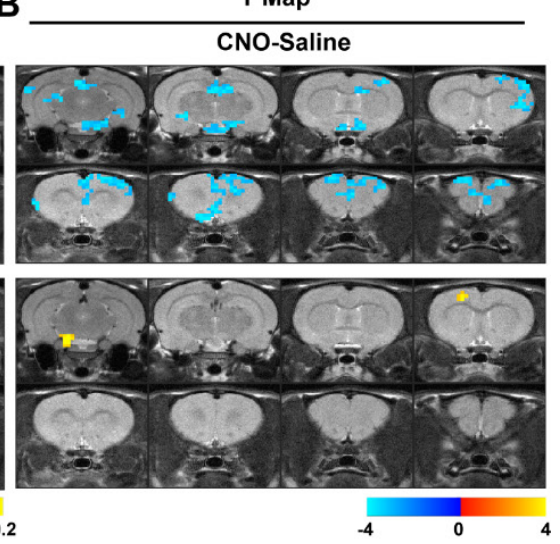

F

Negative Changes
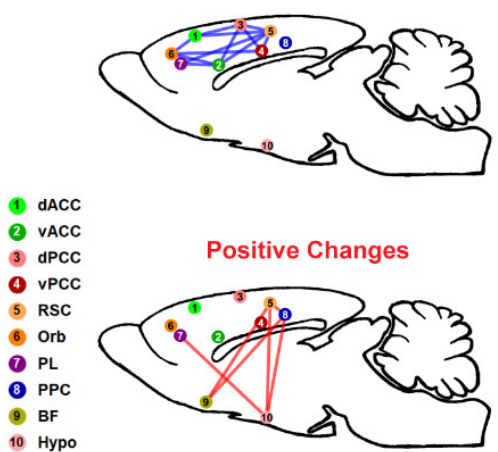

Figure 4. Suppressing $d A C C$ disrupted activity across the DMN. A) DMN constructed using fractional amplitude of low-frequency fluctuations (fALFF) after saline and CNO injections; B) Voxel-wise fALFF changes after DREADD inhibition $(p<0.05$, linear mixed model, family-wise error corrected); C) ROI-wise fALFF changes after DREADD inhibition $\left({ }^{*}: p<0.05 ;{ }^{* *}: p<0.01 ;{ }^{* * *}: p<0.005 ; \#: p=0.11\right)$. D) RSFC between DMN regions in the DREADD and sham groups after saline and CNO injections, respectively; E) RSFC changes between DMN nodes after dACC inhibition $(p<0.05$, linear mixed model, FDR corrected); F) Negative and positive RSFC changes after dACC inhibition $(p<0.05$, linear mixed model, FDR corrected). 

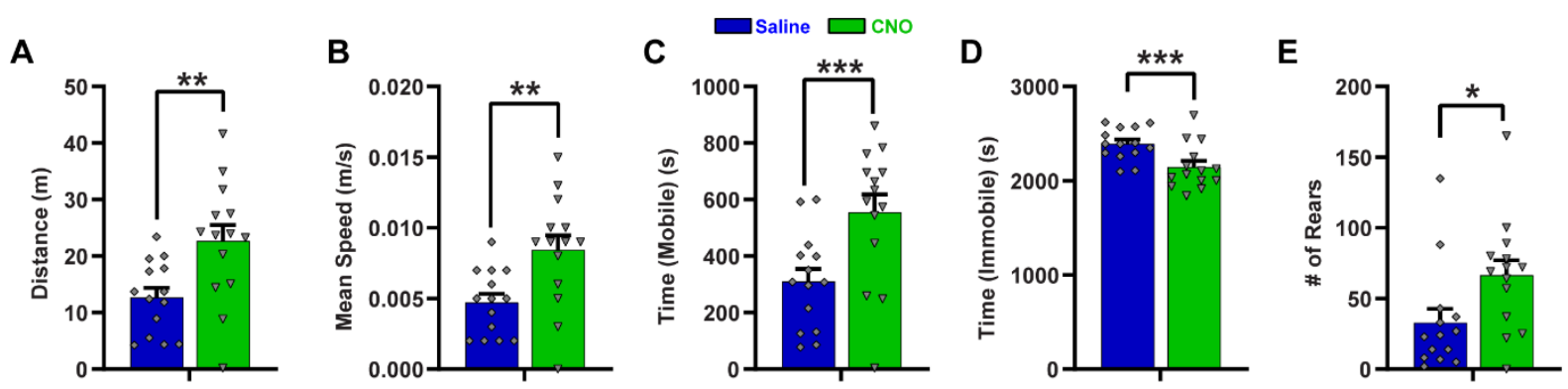

$\mathbf{F}$

dPCC
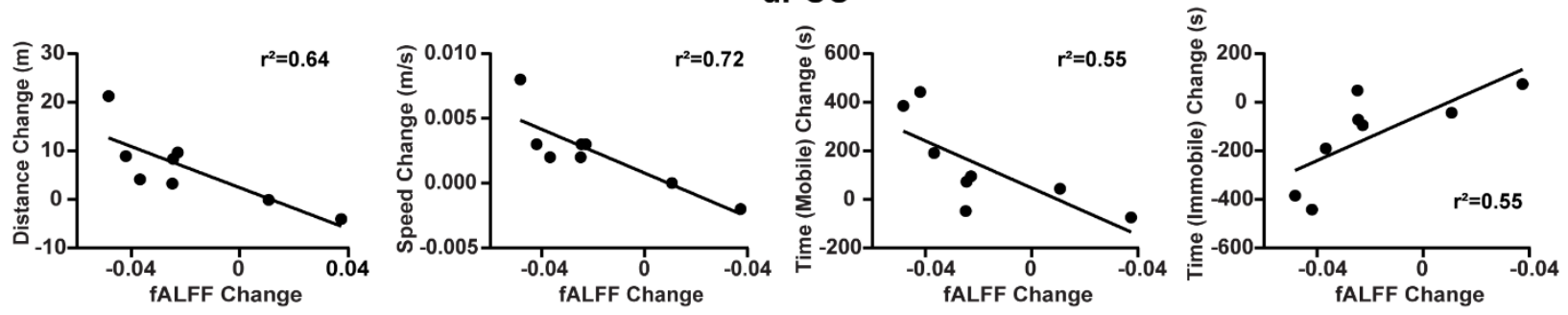

G
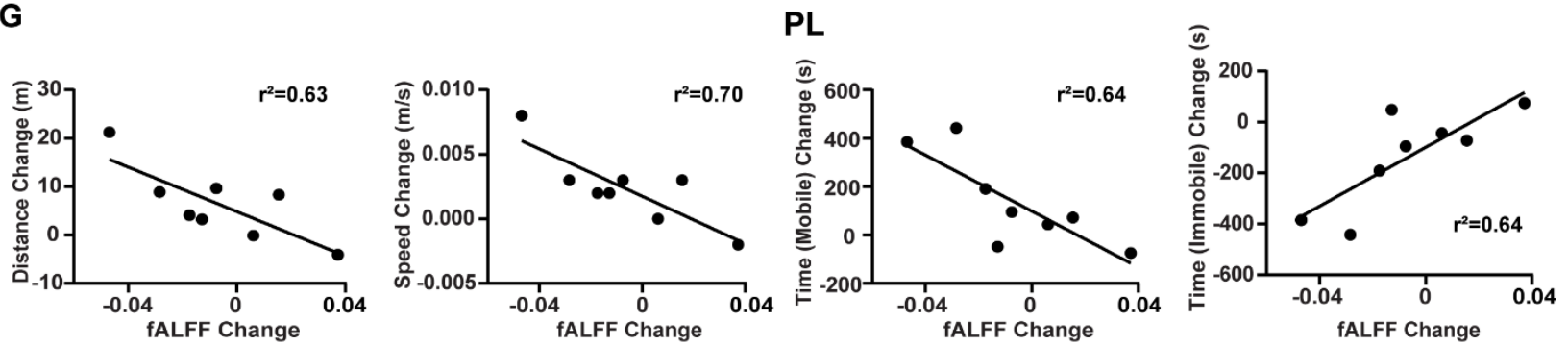

Figure 5. Suppressing $d A C C$ changed $D M N$-related behavior ( $n=14)$. A) Total distance traveled in homecage (45 min); B) Mean speed; C) Total mobile time; D) Total immobile time for at least 2s. E) Total rearing behavior. ${ }^{*}: p<0.05 ;{ }^{* * *}: p<0.005$. F) Correlations of dPCC activity and DMN-related behaviors. G) Correlations of PL activity and DMNrelated behaviors. 

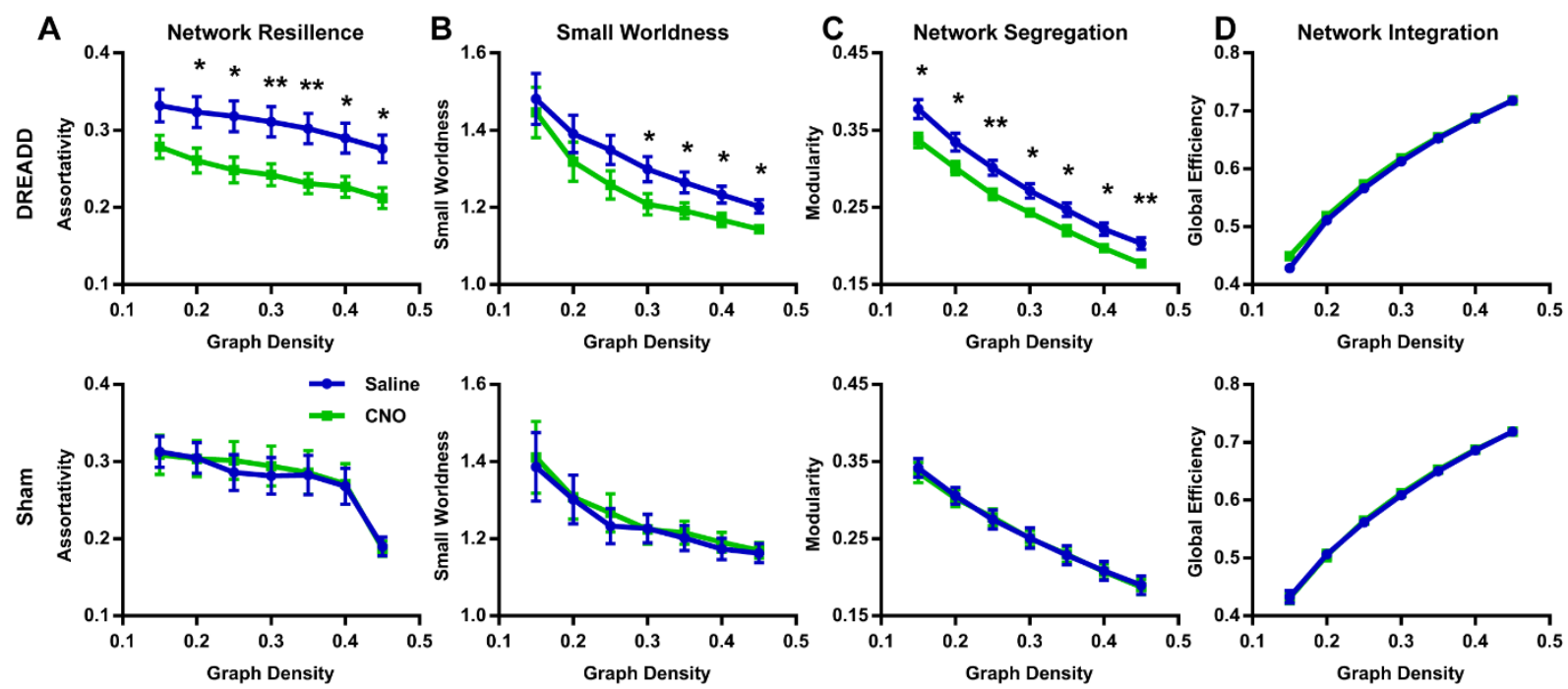

771 Figure 6. Suppressing a hub node changed whole-brain network topology. A) Network

772 resilience; B) Small-worldness; C) Network segregation; D) Network integration. Linear

773 mixed model; DREADD: $n=19,78$ scans; sham: $n=8,41$ scans. ${ }^{*}: p<0.05$; ${ }^{* *}: p<0.01$. 
A

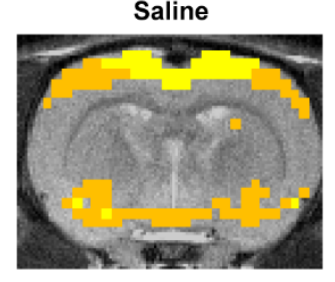

CNO

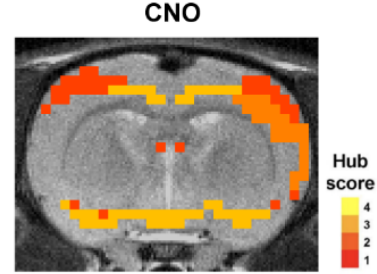

B

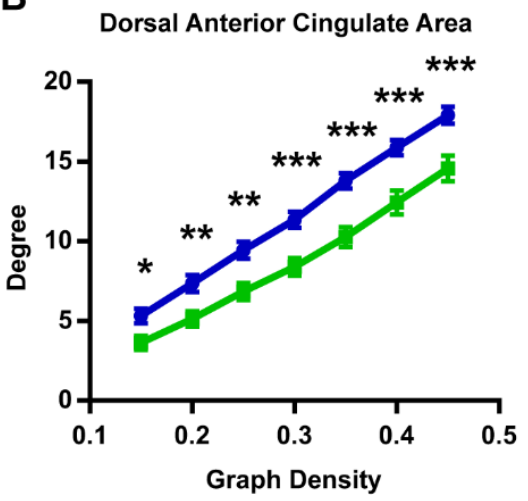

C

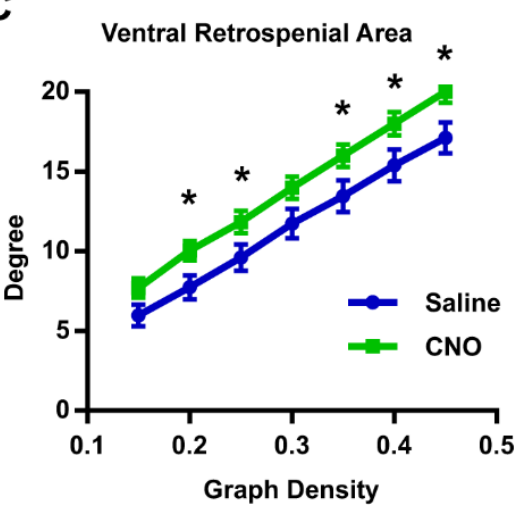

Figure 7. New hub emerged after the inactivation of an existing hub. A) Hub scores 777 without and with dACC inactivation, respectively; B) The degree of dACC and ventral 778 RSC without and with dACC inactivation (linear mixed model; $n=19,78$ scans). *: $p<$ $7790.05 ;{ }^{* *}: p<0.01 ;{ }^{* * *}: p<0.005$. 

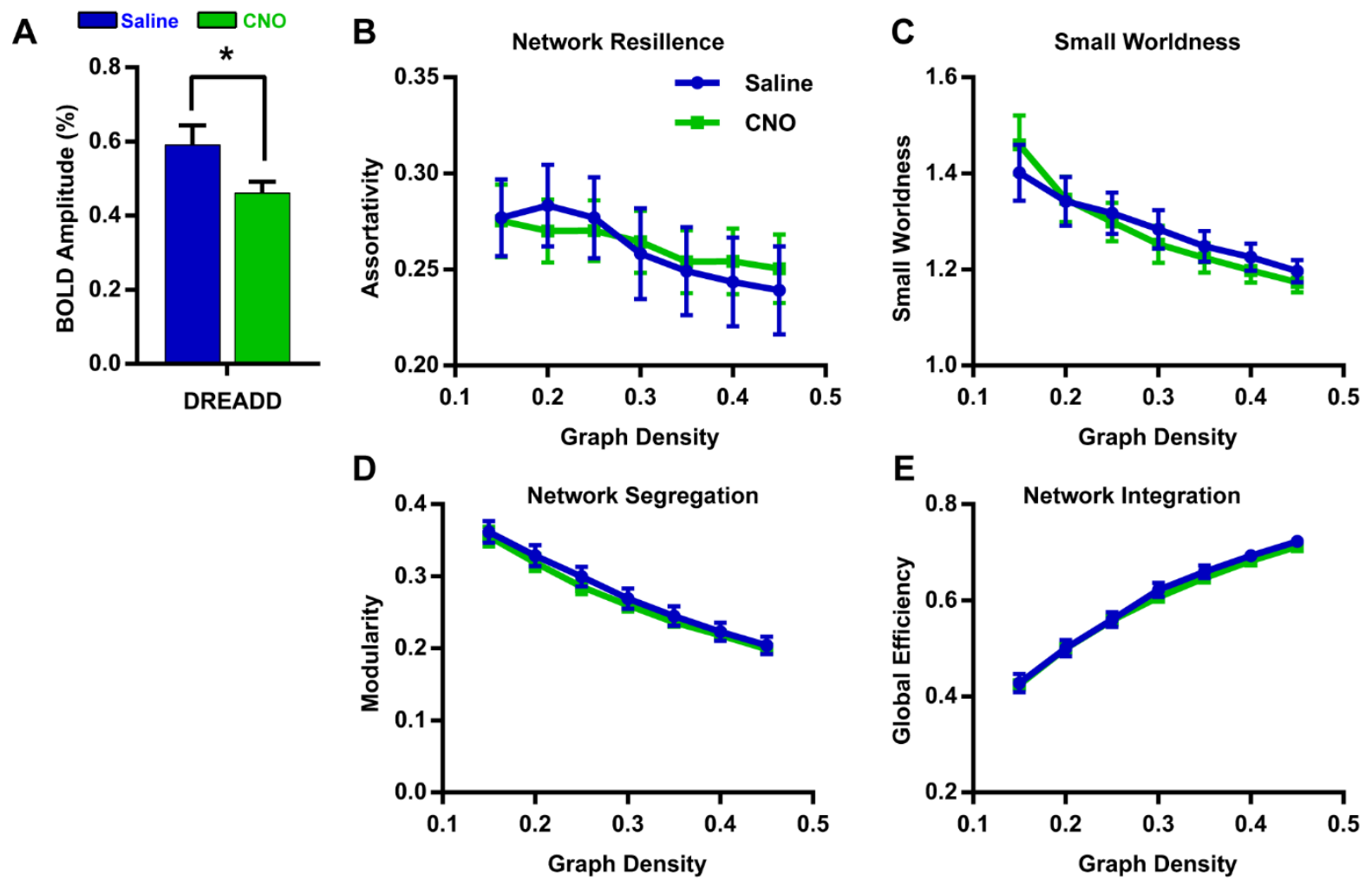

Figure 8. Suppressing a non-hub node did not change whole-brain network topology. A) 783 Amplitude of spontaneous BOLD activity in the primary visual cortex after CNO and saline injections ( $p<0.05, n=8,66$ scans); B) Network resilience; C) Small-worldness; D) Network segregation; E) Network integration. 


\title{
How brain reacts to targeted attack at a hub region
}

\author{
Wenyu $\mathrm{Tu}^{1}$, Zilu $\mathrm{Ma}^{2}$, Yuncong $\mathrm{Ma}^{2}$ and Nanyin Zhang ${ }^{1,2 *}$
}

${ }^{1}$ Neuroscience Program, The Huck Institutes of the Life Sciences, The Pennsylvania State University, University Park, PA 16802, USA

${ }^{2}$ Department of Biomedical Engineering, The Pennsylvania State University, University Park, PA 16802, USA

\section{*Address for correspondence:}

Nanyin Zhang, PhD

Professor of Biomedical Engineering and Electrical Engineering

Lloyd \& Dorothy Foehr Huck Chair in Brain Imaging

The Huck Institutes of the Life Sciences

The Pennsylvania State University

W-341 Millennium Science Complex, University Park, PA 16802, USA

Email: nuz2@psu.edu

\section{Supplementary Information}


A

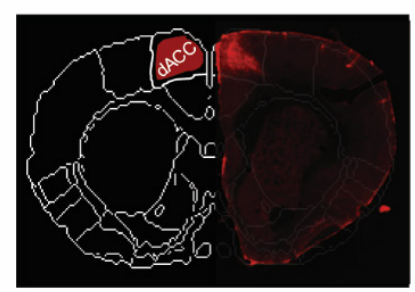

B

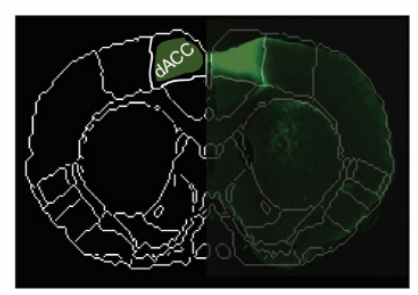

C

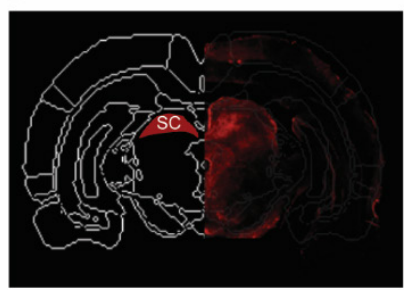

Figure S1. Representative Histology. Protein expression is shown in the right hemisphere. A) DREADD expression in the dorsal anterior cingulate cortex (dACC); B) GFP in the dACC (i.e. sham rats); C) DREADD in the superior colliculus. Anatomical definition is shown in the left hemisphere. 

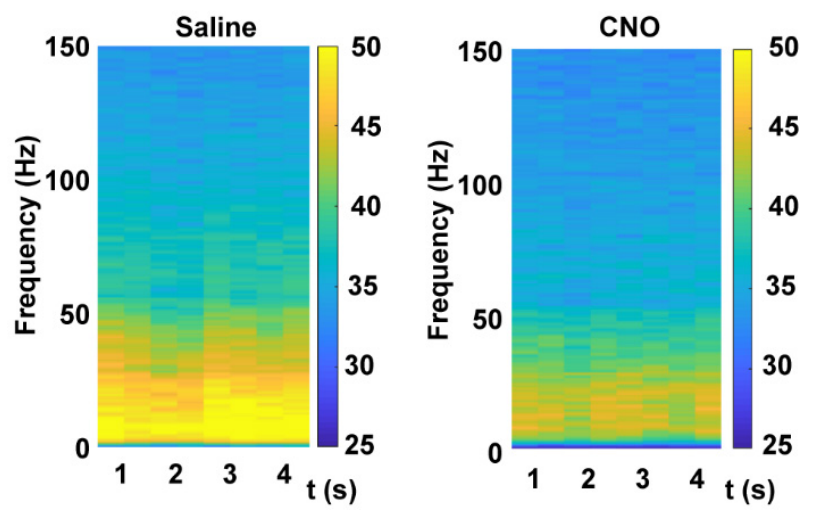

Figure S2. LFP spectrograms after saline and CNO injections in a representative rat with $D R E A D D$ s infused in the SC. 

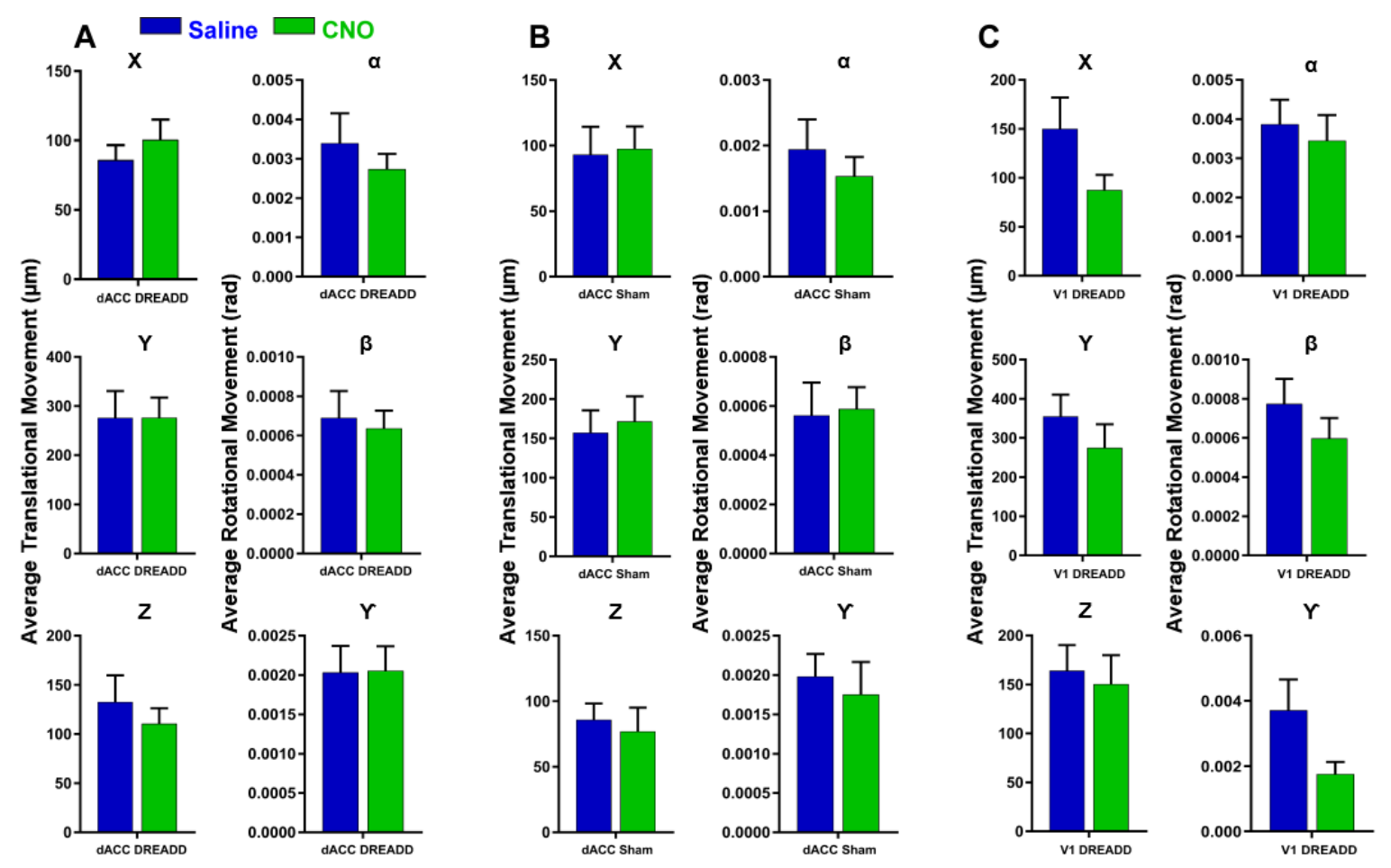

Figure S3. Motion levels in all groups. A. dACC DREADD ( $n=19,78$ scans); B. dACC sham ( $n=8,41$ scans); C. V1 DREADD ( $n=8,66$ scans). Left column: translational movement (in $\mu \mathrm{m}$ ). Right column: rotational movement (in rad) (linear mixed model, $p>$ 0.05 for all 6 parameters in all groups). 
A

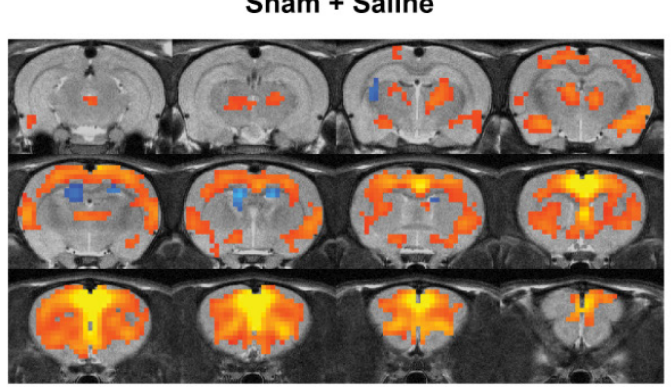

B

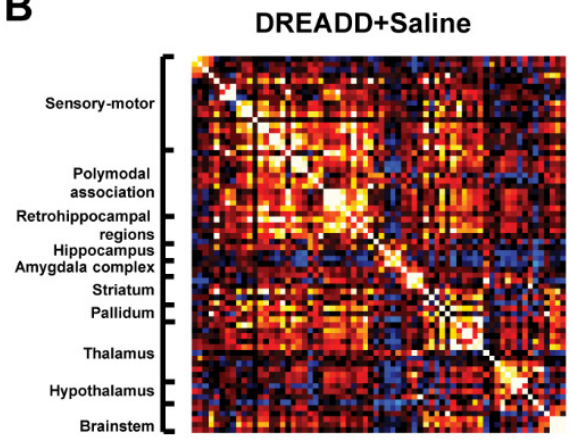

C

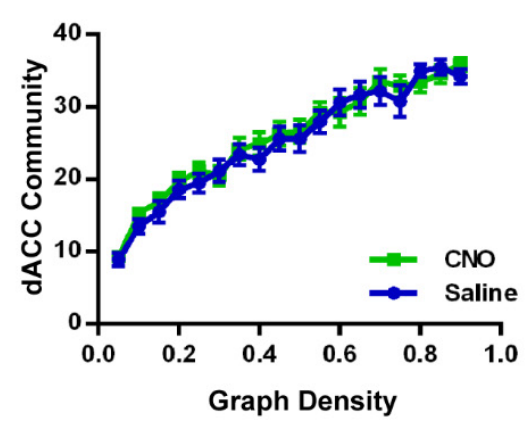

Sham + CNO

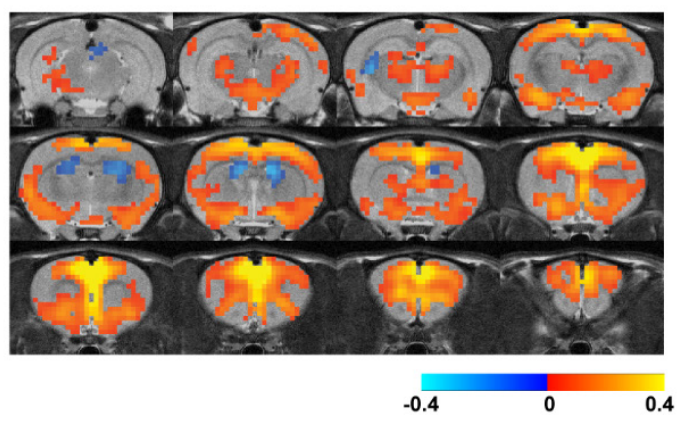

DREADD+CNO

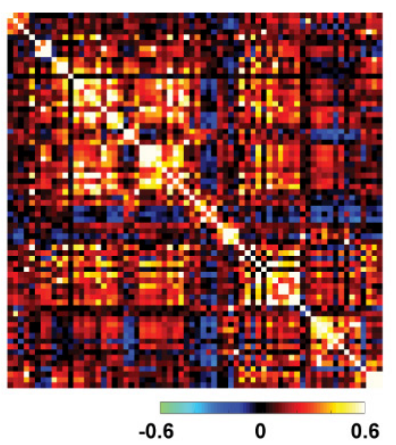

T Map

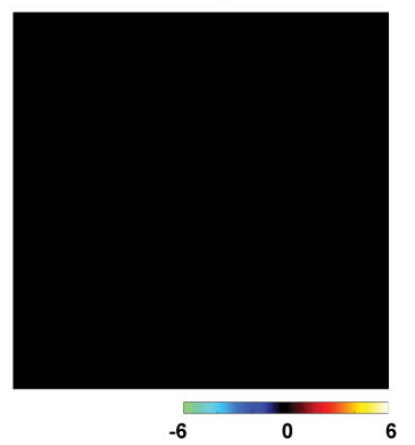

Figure S4. Sham rats did not show reduced RSFC or dACC community size. A) Averaged seedmaps after CNO and saline injections in the sham group (one sample t test, linear mixed model, $p<0.0005$, FDR corrected; n=8, 41scans); B) Averaged ROI-wise RSFC matrices after saline and CNO injections in the sham group. No connections exhibit RSFC changes (two-sample t-tests, linear mixed model, $p<0.05$, FDR corrected). C) The size of the dACC-associated community as a function graph density after saline and CNO injections in the sham group (two-sample t-tests, linear mixed model, $p>0.05$ for all densities tested). 
Saline

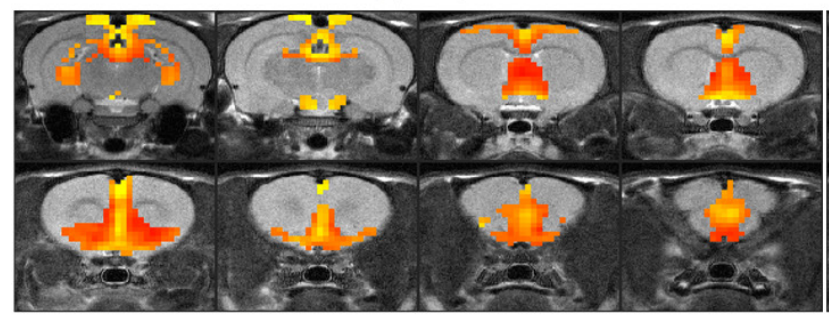

1
CNO

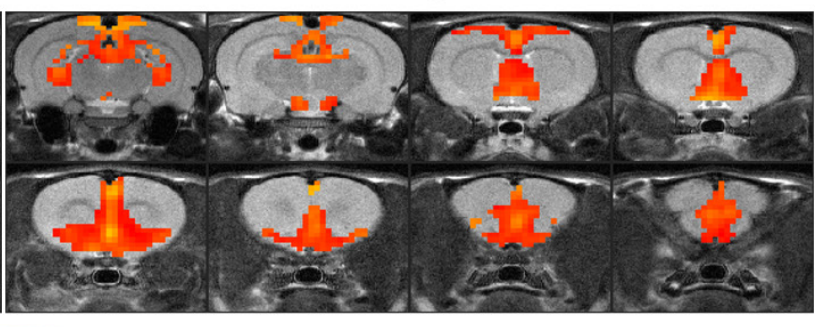

5

Figure S5. DMN pattern revealed by independent component analysis. rsfMRI data collected after saline and CNO injections were pooled together, and Group ICA was conducted. The DMN component was back projected to each group after group ICA. 

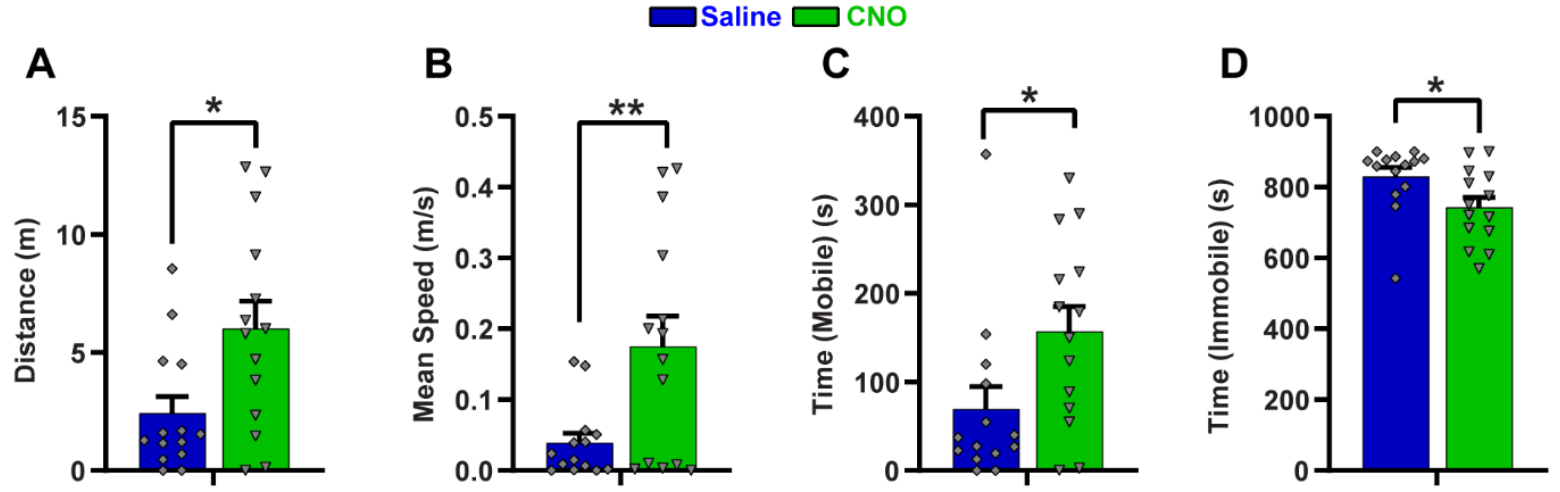

Figure S6. Suppressing dACC changed DMN-related behavior $(n=14)$. A) Total distance traveled; B) Mean speed; C) Total mobile time; and D) Total time when the animal was immobile for at least $2 \mathrm{~s}$ in home-cage in the last $15 \mathrm{~min}$ of tests (i.e. $30 \mathrm{~min}$ after injection of CNO or Saline). ${ }^{*}: p<0.05,{ }^{* *}: p<0.01$. 

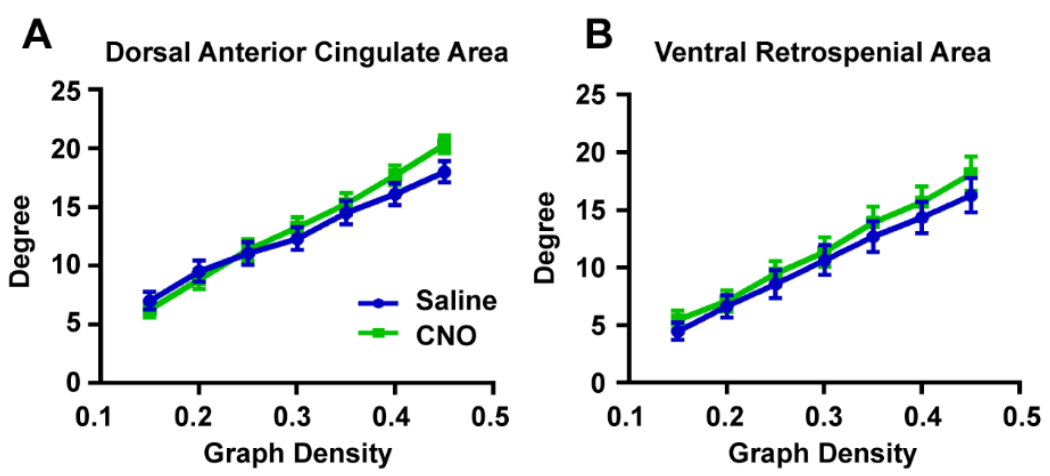

Figure S7. Sham group did not show changed degree of dACC or RSC. Degree changes of the dACC and ventral RSC in the sham group (two-sample t-tests, linear mixed model, $p>0.05$ for all densities tested, $n=8,41$ scans). 

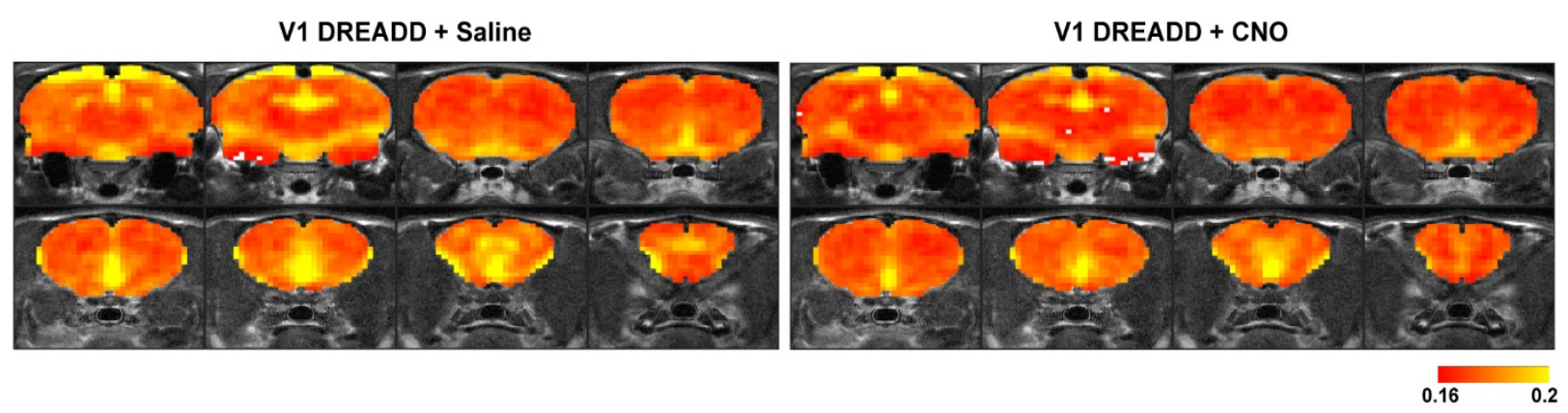

Figure S8. Suppressing V1 did not affect DMN activity. DMN constructed using fALFF in the V1 DREADD group after saline and CNO injections ( $n=8,66$ scans). 


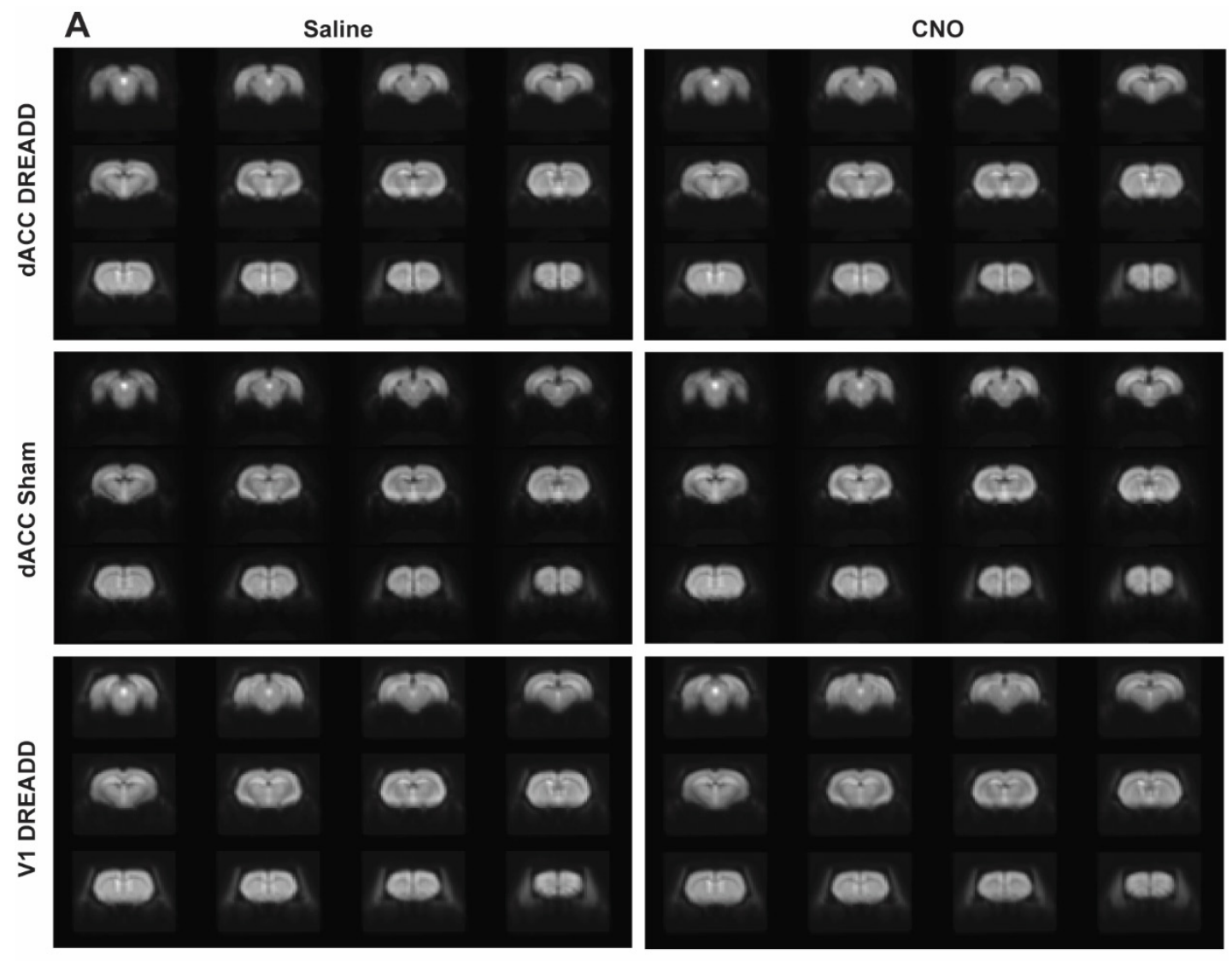

B

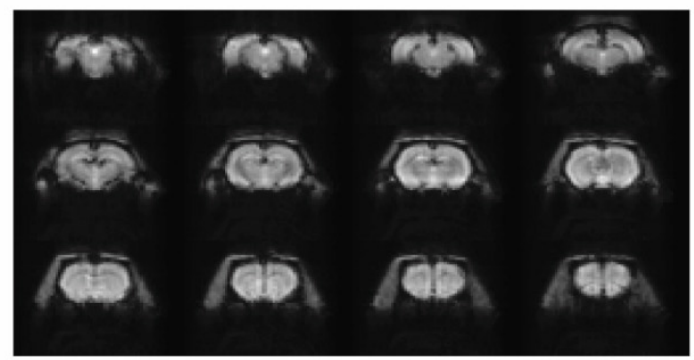

Figure S9. Raw EPI data. A. Averaged EPI image for each group and condition (dACC DREADD; dACC Sham; V1 DREADD); B. Example of a single EPI volume. 